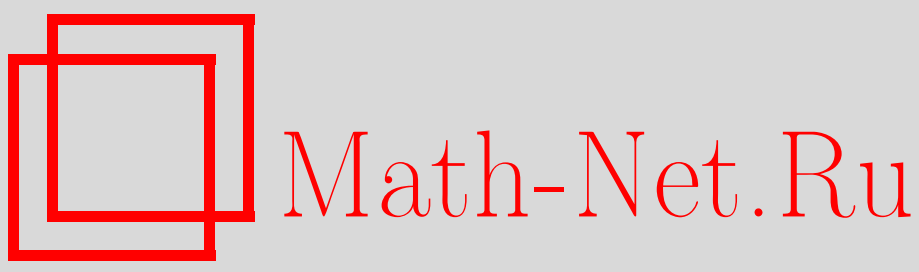

А. В. Пухликов, Бирационально жесткие многообразия с пучком двойных накрытий Фано. I, Матем. сб., 2004, том 195, номер 7, 127-160

DOI: https://doi.org/10.4213/sm837

Использование Общероссийского математического портала Math-Net.Ru подразумевает, что вы прочитали и согласны с пользовательским соглашением http://www . mathnet.ru/rus/agreement

Параметры загрузки:

IP: 3.85 .7 .115

26 апреля 2023 г., $17: 47: 18$ 
УДК 513.6

\author{
А.В. Пухликов
}

\title{
Бирационально жесткие многообразия с пучком двойных накрытий Фано. I
}

\begin{abstract}
Доказано, что общее расслоение Фано $\pi: V \rightarrow \mathbb{P}^{1}$, слой которого - двойная гиперповерхность Фано индекса 1, является бирационально сверхжестким, если выполнено условие достаточной закрученности по базе. В частности, на многообразии $V$ нет других структур расслоения на рационально связные многообразия. Доказательство получено методом максимальных особенностей.

Библиография: 30 названий.
\end{abstract}

\section{§ 0. Введение}

Предметом изучения в настоящей статье является бирациональная геометрия многомерных алгебраических многообразий с пучком двойных накрытий Фано. Основной результат работы - теорема о бирациональной жесткости этих многообразий при условии достаточной закрученности по базе - сформулирован ниже, в п. 0.3. В статье представлены результаты первого этапа исследования; вторая часть работы, посвяшенная ослаблению условия закрученности, будет опубликована в последующей статье.

0.1. Бирационально жесткие многообразия. Рационально связное проективное многообразие $V$ с $\mathbb{Q}$-факториальными терминальными особенностями называется бирационально жсестким, если для любого бирационального отображения

$$
\chi: V \rightarrow V^{\prime}
$$

где $V^{\prime}$ - многообразие из того же класса, и любой подвижной линейной системы $\Sigma^{\prime}$ на $V^{\prime}$ сушествует бирациональный автоморфизм $\chi^{*} \in \operatorname{Bir} V$, обеспечивающий выполнение неравенства

$$
c(\Sigma) \leqslant c\left(\Sigma^{\prime}\right)
$$

где $\Sigma=\left(\chi \circ \chi^{*}\right)_{*} \Sigma^{\prime}-$ собственньй прообраз линейной системы $\Sigma^{\prime}$ на $V$ относительно бирационального отображения

$$
\chi \circ \chi^{*}: V \stackrel{\chi^{*}}{\rightarrow} V \stackrel{\underset{\chi}{\chi}}{\rightarrow} V^{\prime}
$$

а символ $c(\cdot)$ обозначает порог канонического присоединения линейной системы $|\cdot|$,

$$
c(\Lambda)=\sup \left\{\varepsilon \in \mathbb{Q}_{+} \mid D+\varepsilon K \in A_{+}^{1}(\cdot)\right\}
$$

Работа выполнена в рамках Программы поддержки молодых российских ученых-докторов наук (грант № МД-328.2003.01) и при поддержки Фонда содействия отечественной науке.

(C) А. В. Пухликов 2004 
где $D \in \Lambda$ - произвольный дивизор линейной системы $\Lambda, K$ - канонический класс многообразия, $A_{+}^{1}(\cdot) \subset A^{1}(\cdot) \otimes \mathbb{R}$ - замкнутьй конус эффективных классов на рассматриваемом многообразии. Многообразие $V$ называется бирачионально сверхжестким, если неравенство (1) выполнено всегда при $\chi^{*}=\mathrm{id}_{V}$.

Пусть $\pi: V \rightarrow \mathbb{P}^{1}$ - расслоение на рационально связные многообразия, где $V$ имеет $\mathbb{Q}$-факториальные терминальные особенности. (Согласно теореме Грабера-Харриса-Старра [1] в этом случае само многообразие $V$ автоматически рационально связно.) Следующий вопрос является ключевьм для понимания бирациональной геометрии многообразия $V$ :

существуют ли на $V$ другие (т.е. отличные от исходной $\pi: V \rightarrow \mathbb{P}^{1}$ ) структуры расслоения на рационально связные многообразия?

Предположим, что $V$ неособо, $\operatorname{Pic} V=\mathbb{Z} K_{V} \oplus \pi^{*} \operatorname{Pic} \mathbb{P}^{1}$ и выполнено следуюшее условие:

$$
-K_{V} \notin \operatorname{Int} A_{+}^{1} V \text {. }
$$

Пусть $F_{t}=\pi^{-1}(t)$ - слой над точкой $t \in \mathbb{P}^{1}, F \in$ Pic $V-$ класс слоя. Условие (2) означает, что если $D \sim-n K_{V}+l F-$ эффективный дивизор на $V$, то $l \in \mathbb{Z}_{+}$. Условия этого типа для трехмерных расслоений Мори обсуждаются в [2]. Следующий факт хорошо известен (см. [3; с. 20-22]).

ПРЕДЛОЖЕНИЕ 0.1. В сделанных выше предположениях пусть $V$ - бираиионально сверхжесткое многообразие. Тогда:

(i) на $V$ имеется единственная нетривиальная структура расслоения на рационально связные многообразия - морфизм $\pi$; иными словами, если $\tau: W \rightarrow T-$ расслоение на рационально связные (или унилинейчатые) многообразия и $\chi: V \rightarrow W-$ бирациональное отображение, то $\chi$ переводит слои в слои, т.е. имеет место коммутативная диаграмма

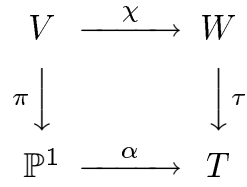

для некоторого отображсения $\alpha: \mathbb{P}^{1} \rightarrow T$;

(ii) если $\tau: W \rightarrow T=\mathbb{P}^{1}$ - другое расслоение того жсе типа, т.е. Pic $W=$ $\mathbb{Z} K_{W} \oplus \tau^{*} \operatorname{Pic} \mathbb{P}^{1}$ и $\chi: V \rightarrow W$ (послойное) бирачиональное отображсние, то ұ есть изоморфизм слоев общего положсения.

Таким образом, свойство бирациональной жесткости сводит бирациональную геометрию многообразия $V$ к бирегулярной геометрии расслоения $V / \mathbb{P}^{1}$. Этим и объясняется выбор слова "жесткость": многообразие $V$ не допускает бирациональных перестроек внутри естественного класса расслоений Фано с относительным числом Пикара единица.

В настоящее время известно большое число классов бирационально (сверх)жестких многообразий Фано (см., например, [4]-[10]). Имеющиеся примеры позволяют предположить, что свойство (сверх)жесткости является типичным в размерности 3 и выше. О расслоениях Фано известно меньше, их бирациональную геометрию изучать труднее. Краткую историю теории бирациональной жесткости для 
расслоений Фано см. ниже, в п. 0.4. Цель настоящей работы - доказать бирациональную сверхжесткость расслоений $V / \mathbb{P}^{1}$, слои которых суть двойные гиперповерхности Фано индекса 1 [7].

0.2. Многообразия с пучком двойных накрытий. Символом $\mathbb{P}$ обозначаем проективное пространство $\mathbb{P}^{M+1}$ над полем комплексных чисел $\mathbb{C}$. Пусть

$$
\mathscr{G}=\mathbb{P}\left(H^{0}\left(\mathbb{P}, \mathscr{O}_{\mathbb{P}}(m)\right)\right),
$$

$3 \leqslant m \leqslant M-1,-$ пространство всех гиперповерхностей Фано степени $m, \mathscr{W}-$ пространство всех гиперповерхностей степени $2 l$ в $\mathbb{P}$, причем $m+l=M+1$. Пусть

$$
\mathscr{F}=\{F \mid \sigma: F \stackrel{2: 1}{\longrightarrow} G\}
$$

- класс алгебраических многообразий, двулистно накрьвающих гиперповерхности $G \in \mathscr{G}$ с ветвлением над $W \cap G, W \in \mathscr{W}$, и пусть $\mathscr{F}_{\text {sm }} \subset \mathscr{F}$ - открытое множество гладких двойных гиперповерхностей, соответствующих парам $(G, W \cap G)$ гладких многообразий. Очевидно, $F \in \mathscr{F}_{\mathrm{sm}}$ - гладкое многообразие Фано индекса 1 с группой Пикара $\mathrm{Pic} F=\mathbb{Z} K_{F}$. Пусть $\mathscr{F}_{\mathrm{sm}}^{\mathrm{reg}} \subset \mathscr{F}_{\mathrm{sm}}-$ открытое подмножество многообразий $F \in \mathscr{F}_{\mathrm{sm}}$, удовлетворяющих условию регулярности в пп. 1.3, 1.4 ниже (идентичному условию регулярности п. 1.3 в [7]). Напомним, что в [7] был доказан следующий факт.

Tеорема А. (i) Любое многообразие $F \in \mathscr{F} \mathrm{sm}$ бирационально сверхжесткое.

(ii) Множество $\mathscr{F}_{\mathrm{sm}}^{\mathrm{reg}}$ непусто. Более того, имеет место неравенство

$$
\operatorname{codim}_{\mathscr{F}_{\mathrm{sm}}}\left(\mathscr{F}_{\mathrm{sm}} \backslash \mathscr{F}_{\mathrm{sm}}^{\mathrm{reg}}\right) \geqslant 2 .
$$

Положим $\mathscr{F}_{\text {sing }}=\mathscr{F} \backslash \mathscr{F}_{\text {sm }}, \operatorname{codim}_{\mathscr{F}} \mathscr{F}_{\text {sing }}=1$. Пусть $\mathscr{F}_{\text {sing }}^{\mathrm{reg}}-$ открытое в $\mathscr{F}_{\text {sing }}$ подмножество особых двойных гиперповерхностей, удовлетворяющих условию регулярности пп. 1.3, 1.4 ниже. Как отмечено в пп. $1.3,1.4$, имеет место неравенство

$$
\operatorname{codim}_{\mathscr{F}}\left(\mathscr{F}_{\text {sing }} \backslash \mathscr{F}_{\text {sing }}^{\mathrm{reg}}\right) \geqslant 2 .
$$

В настоящей работе изучаются расслоения Фано $V / \mathbb{P}^{1}$, каждый слой $F_{t}=\pi^{-1}(t)$, $t \in \mathbb{P}^{1}$, которых есть многообразие из семейства $\mathscr{F}$. Положим

$$
\mathscr{F}^{\mathrm{reg}}=\mathscr{F}_{\mathrm{sm}}^{\mathrm{reg}} \cup \mathscr{F}_{\mathrm{sing}}^{\mathrm{reg}} .
$$

В силу сказанного выше $\operatorname{codim}_{\mathscr{F}}\left(\mathscr{F} \backslash \mathscr{F}^{\mathrm{reg}}\right) \geqslant 2$. Поскольку расслоение $V / \mathbb{P}^{1}$ можно интерпретировать как морфизм $\mathbb{P}^{1} \rightarrow \mathscr{F}$, т.е. кривую в $\mathscr{F}$, для обшего многообразия $V / \mathbb{P}^{1}$ имеем

$$
F_{t} \in \mathscr{F}^{\mathrm{reg}}
$$

для любого $t \in \mathbb{P}^{1}$. В этом случае назовем расслоение $V / \mathbb{P}^{1}$ регулярныц. Конструкция регулярных расслоений $\Phi$ ано $V / \mathbb{P}^{1}$ описана ниже в п. 1.2. 


\section{3. Формулировка основного результата.}

ТЕОРема 1. Предположим, что регулярное расслоение $V / \mathbb{P}^{1}$ удовлетворяет $K^{2}$-условию:

$$
K_{V}^{2} \notin \operatorname{Int} A_{+}^{2} V
$$

әде $A_{+}^{2} V \subset A^{2} V \otimes \mathbb{R}$ - замкнутый конус әффективных ииклов коразмерности два. Тогда расслоение $V / \mathbb{P}^{1}$ бирационально сверхжесткое.

Символ $A^{i} \#$ обозначает, как обычно, группу классов циклов коразмерности $i$ многообразия \# по модулю численной эквивалентности.

СлеДСТВИЕ 0.1. (i) Для расслоения $V / \mathbb{P}^{1}$ общего положения имеет место равенство

$$
\operatorname{Bir} V=\operatorname{Aut} V=\mathbb{Z} / 2 \mathbb{Z}=\{\operatorname{id} \tau\},
$$

где $\tau \in$ Aut $V$ - инволюиия Галуа двойного накрытия $V / Q$.

(ii) Многообразие $V$ нерационально.

Часть (i) вытекает из предложения 0.1 и теоремы А. Часть (ii) очевидна.

Доказательство теоремы 1 будет проведено в несколько шагов. Ниже в п. 1.1 сформулировано достаточное условие бирациональной сверхжесткости произвольного расслоения $\Phi$ ано $V / \mathbb{P}^{1}$ в терминах численной геометрии слоев (теорема 2). Этот факт был по существу доказан в [3], [11], хотя в упомянутых работах речь шла о расслоениях Фано конкретного вида. Здесь мы не будем повторять эти рассуждения, отсылая читателя к [3], [11].

Теперь для доказательства теоремы 1 необходимо проверить, что слои расслоения $V / \mathbb{P}^{1}$, т.е. регулярные двойные гиперповерхности Фано индекса 1 (в смысле условий регулярности, сформулированных ниже в пп. 1.3, 1.4), удовлетворяют условиям теоремы 2. Эта проверка и составляет наше доказательство. Она проводится в $\S \S 2$ и 3. Бирациональная геометрия многообразий с пучком двойных накрытий Фано, не удовлетворяющим $K^{2}$-условию, будет изучена в следующей статье-второй части работы.

ЗАмеЧАнИЕ. Сверхжесткость расслоений Фано $V / \mathbb{P}^{1}$, слои которых - двойные пространства $(m=1)$ и двойные квадрики $(m=2)$ индекса 1 , доказана в [3] и [12], [13] соответственно, поэтому в настоящей работе эти многообразия не рассматриваются.

0.4. Исторические замечания. Описание структур расслоения на рационально связные (унилинейчатые) многообразия - очень старая тема. Классическое доказательство теоремы Нётера о группе Кремоны плоскости, изложенное Ю.И. Маниным в [14], можно рассматривать в этом ключе: шаг за шагом модифицируется некоторый пучок рациональных кривых на $\mathbb{P}^{2}$, т.е. происходит переход от одной структуры $\mathbb{P}^{1}$-расслоения на плоскости к другой. Изучать бирациональную геометрию многообразий размерности выше двух, на которых имеется много различных структур рационально связного расслоения, очень трудно. Кроме нескольких исключительных типов, такие многообразия все еще недоступны для современной техники. Однако в некотором смысле общие рационально связные многообразия обладают лишь одной структурой рационально связного расслоения с условием минимальности типа расслоения Фано с относительньм числом Пикара единица. Это и есть феномен бирациональной жесткости. 
В современной бирациональной геометрии бирационально жесткие многообразия впервые появляются в работах Ю.И. Манина в виде поверхностей дель Пещо над незамкнутыми полями [15], [16]. Первые теоремы о бирациональной жесткости нетривиальных рационально связных расслоений - это теоремы В. А. Исковских о единственности пучка рациональных кривых для поверхностей над незамкнутыми полями [17], [18], продолжающие работы Ю. И. Манина. Изучение абсолютного и относительного случаев в размерности два над незамкнутым полем подготовило почву для работы в высших размерностях.

После того как в классической работе В. А. Исковских и Ю.И. Манина [5] была развита техника пробного класса, позволившая доказать (в современной терминологии) бирациональную сверхжесткость гладкой трехмерной квартики $V_{4} \subset \mathbb{P}^{4}$ (фактически в [5] была также доказана сверхжесткость двойного пространства с ветвлением в секстике и сделан решающий шаг в доказательстве бирациональной жесткости двойной квадрики индекса 1 [19]), были предприняты попытки с помощью этой техники получить аналогичные результаты в относительном варианте, для расслоений над нетривиальной базой. Для одного класса многообразий это удалось сделать сразу во всех размерностях - теорема В.Г. Саркисова доказывает единственность заданной структуры расслоения на коники при условии, что дискриминантный дивизор достаточно велик [20], [21]. Доказательство теоремы В.Г. Саркисова основано на двух технических принципах:

1) технике пробного класса В.А. Исковских и Ю.И. Манина,

2) послойных перестройках.

Возможность послойно перестраивать расслоения на коники, оставаясь при этом в классе гладких многообразий, является уникальным свойством этих многообразий. В каком-то смысле указанная особенность проистекает из того, что группа автоморфизмов слоя Aut $\mathbb{P}^{1}$ очень велика: скажем, для типичного многообразия Фано размерности выше двух эта группа конечна, так что нет надежды использовать аналогичные соображения для многомерных расслоений Фано.

После опубликования работ Саркисова [20], [21] единственным классом трехмерных рационально связных многообразий, о бирациональной геометрии которых почти ничего не было известно, оставались расслоения на поверхности дель Пеццо над $\mathbb{P}^{1}$. Попытки использовать послойные перестройки по аналогии с теоремой Саркисова оказались безуспешными, так как сразу выводили за пределы класса гладких многообразий и, более того, приобретаемые особенности оказывались неконтролируемыми. Но и вышеупомянутая техника пробного класса отказывалась работать. Многолетние (середина 80x - середина 90x) попытки построить хотя бы единичные примеры трехмерных расслоений дель Пещо, аналогичных жестким расслоениям на коники Саркисова, не увенчались успехом. Эта деятельность подытожена в [22]: единственным результатом почти десятилетней работы и огромного количества проделанных вычислений оказались гипотезы - и никакого существенного продвижения в их доказательстве. Как видно из [22], отсутствовало понимание, почему техника пробного класса, столь безупречно работающая в абсолютном случае (трехмерная квартика [5]), не позволяет продвинуться ни на шаг в случае расслоений дель Пещо: пробный класс просто не удавалось построить. 
Положение радикально изменилось с появлением статьи [3]. Попутно сразу объяснилось, почему техника пробного класса не обобщалась на относительньй случай: как оказалось, искомого класса просто не существует. Для трехмерной квартики техника пробного класса эквивалентна введенной в [3], [6], [8] технике подсчета кратностей. Однако техника подсчета кратностей гораздо более гибкая, потому что описьвает свойства некоторого эффективного цикла коразмерности 2 (самопересечения подвижной линейной системы, задающей бирациональное отображение), а не единственное число - индекс пересечения этого цикла с пробным классом. В относительном случае, когда база расслоения нетривиальна, эффективный цикл раскладывается на горизонтальную и вертикальную компоненты. Грубо говоря, для каждой из этих компонент нужен свой пробный класс.

После появления работы [3] развитые в ней методы были использованы для доказательства бирациональной жесткости больших классов многомерных расслоений Фано [11]-[13]. В этих работах (как и в настоящей статье) бирациональная жесткость выводилась из $K^{2}$-условия. Однако методы этих работ сохраняют силу и при некотором ослаблении $K^{2}$-условия и позволяют полностью описать бирациональную геометрию многообразия, см. [23], [24] и серию статей [25]-[27], в которых доказана бирациональная жесткость нескольких классов трехмерных расслоений дель Пещцо над $\mathbb{P}^{1}$, не рассмотренных в [3] из-за невыполнения $K^{2}$-условия. Однако при слишком сильном отклонении от $K^{2}$-условия многообразие становится недоступным для изучения.

Отметим также, что попытки сколько-нибудь улучшить результаты Саркисова или доказать критерий рациональности для расслоений на коники до сих пор безуспешны [28], несмотря на прогресс в общей теории факторизации бирациональных отображений между трехмерными расслоениями Мори (программа Саркисова [29], [30]). По этому поводу см. недавнюю статью [2]; к ее обсуждению мы вернемся в последующих работах.

0.5. Благодарности. Часть работы (соответствующая второму параграфу настоящей статьи) была проделана автором в Байройтском университете в 2001 г. в период пребывания в Байройте в качестве Гумбольдтовского стипендиата. Ключевой шаг (анализ особенностей, связанных с двойной точкой на дивизоре ветвления слоя, составляющий содержание третьего параграфа статьи) был сделан во время работы автора в Институте Макса Планка в Бонне в 2003 г. Автор благодарит Фонд Гумбольдта, Математический институт Байройтского университета (в первую очередь, Т. Петернелла) и Математический институт Макса Планка в Бонне за гостеприимство, прекрасные условия работы и обшую поддержку.

\section{§1. Метод максимальных особенностей и условия регулярности}

1.1. Критерий бирациональной жесткости. Пусть $\pi: V \rightarrow \mathbb{P}^{1}$ - гладкое стандартное расслоение Фано, т.е. $V$ - гладкое многообразие,

$$
\text { Pic } V=\mathbb{Z} K_{V} \oplus \mathbb{Z} F
$$

где $F$-класс слоя. Определим степень горизонтального подмногообразия $Y \subset V$, $\pi(Y)=\mathbb{P}^{1}$, соотношением

$$
\operatorname{deg} Y=\left(Y \cdot F \cdot\left(-K_{V}\right)^{\operatorname{dim} Y-1}\right),
$$


степень вертикального подмногообразия $Y \subset \pi^{-1}(t)-$ соотношением

$$
\operatorname{deg} Y=\left(Y \cdot\left(-K_{V}\right)^{\operatorname{dim} Y}\right)
$$

При таком определении степень самого многообразия $V$ совпадает со степенњю слоя, $\operatorname{deg} V=\operatorname{deg} F$.

Гладкие расслоения Фано, слои которых суть полные пересечения во взвешенных проективных пространствах, обладают также следующим свойством: особенности слоев не более чем нульмерны.

Скажем, что расслоение $Ф$ ано $V / \mathbb{P}^{1}$ удовлетворяет

условию $(\mathrm{v})$, если для любого неприводимого вертикального подмногообразия $Y$ коразмерности $2, Y \subset \pi^{-1}(t)=F_{t}$, и гладкой точки $o \in F_{t}$ выполнено неравенство

$$
\frac{\text { mult }_{o}}{\operatorname{deg}} Y \leqslant \frac{2}{\operatorname{deg} V}
$$

условию (vs), если для любого вертикального подмногообразия $Y \subset F_{t}$ коразмерности 2 (относительно $V$, т.е. простого дивизора на $F_{t}$ ), особой точки $о \in F_{t}$ и бесконечно близкой точки $x \in \widetilde{F}_{t}$, где $\varphi: \widetilde{F}_{t} \rightarrow F_{t}$ - раздутие точки $o, \varphi(x)=o, \widetilde{Y} \subset \widetilde{F}_{t}-$ собственный прообраз подмногообразия $Y$ на $\widetilde{F}_{t}$, вьполнены оценки

$$
\frac{\text { mult }_{o}}{\operatorname{deg}} Y \leqslant \frac{4}{\operatorname{deg} V}, \quad \frac{\operatorname{mult}_{x} \tilde{Y}}{\operatorname{deg} Y} \leqslant \frac{2}{\operatorname{deg} V}
$$

условию (h), если для любого горизонтального подмногообразия $Y$ коразмерности 2 и точки $o \in Y$ выполнена оценка

$$
\frac{\text { mult }_{o}}{\operatorname{deg}} Y \leqslant \frac{4}{\operatorname{deg} V}
$$

Предположим, что $\operatorname{dim} V \geqslant 4$ и многообразие $V$ удовлетворяет условию

$$
A^{2} V=\mathbb{Z} K_{V}^{2} \oplus \mathbb{Z} H_{F}
$$

где $H_{F}=\left(K_{V} \cdot F\right)$ и слой общего положения $F=F_{t} \subset V$ удовлетворяет условию $A^{2} F=\mathbb{Z}\left(H_{F} \cdot H_{F}\right)_{F}$. Положим $A_{\mathbb{R}}^{2} V=A^{2} V \otimes \mathbb{R} \cong \mathbb{R}^{2}$ и определим конус эффективных циклов $A_{+}^{2} V \subset A_{\mathbb{R}}^{2} V$ как замькание (в стандартной топологии) множества

$$
\left\{\lambda \Delta \mid \lambda \in \mathbb{R}_{+}, \Delta-\text { класс эффективного цикла }\right\} .
$$

ОПРЕДЕЛЕНИЕ 1.1. Скажем, что расслоение $\Phi$ ано $V / \mathbb{P}^{1}$ удовлетворяет $K^{2}-y c-$ ловию, если

$$
K_{V}^{2} \notin \operatorname{Int} A_{+}^{2} V
$$


ЗАмечАниЕ. Легко видеть, что $K^{2}$-условие эквивалентно следующему требованию: для любых $a \geqslant 1$ и $b \geqslant 1$ класс

$$
\Delta(a, b)=a K_{V}^{2}-b H_{F}
$$

не является эффективным. В самом деле, $H_{F} \in A_{+}^{2} V$, так что $K_{V}^{2} \in \operatorname{Int} A_{+}^{2} V$ тогда и только тогда, когда $\Delta(N, 1) \in A_{+}^{2} V$ для некоторого $N \geqslant 1$. Отсюда немедленно вытекает эквивалентность двух условий.

Теорема 2. Предположим, что гладжое стандартное расслоение Фано $V / \mathbb{P}^{1}$ удовлетворяет $K^{2}$-условию и условиям (v), (vs) и (h). Тогда $V / \mathbb{P}^{1}-$ бирачионально сверхжесткое расслоение Фано.

Доказательство см. в [3], [11].

1.2. Конструкция расслоения $V / \mathbb{P}^{1}$. Опишем явную конструкцию регулярных расслоений $V / \mathbb{P}^{1}$. Для любого слоя $F \in \mathscr{F}$ reg (особого или гладкого) антиканоническая линейная система $\left|-K_{F}\right|$ задает в точности двойное накрытие $\sigma_{F}: F \rightarrow G \subset \mathbb{P}$. Поэтому $\pi_{*} \mathscr{O}\left(-K_{V}\right)$ есть локально свободный пучок ранга $M+2$ на $\mathbb{P}^{1}$, определяющий локально тривиальное $\mathbb{P}$-расслоение над $\mathbb{P}^{1}$. Многообразие $V$ реализуется как двойное накрытие гладкого дивизора $Q$ на $\mathbb{P}\left(\pi_{*} \mathscr{O}\left(-K_{V}\right)\right)$. Подробнее, пусть

$$
\mathscr{E}=\bigoplus_{i=0}^{M+1} \mathscr{O}_{\mathbb{P}^{1}}\left(a_{i}\right)
$$

- локально свободный пучок, нормализованный таким образом, что $a_{0}=0 \leqslant$ $a_{1} \leqslant \cdots \leqslant a_{i} \leqslant a_{i+1} \leqslant \cdots \leqslant a_{M+1}$. В частности, $\mathscr{E}$ порождается своими сечениями. Пусть $X=\mathbb{P}(\mathscr{E})$ - его Proj в смысле Гротендика, $\pi_{X}: X \rightarrow \mathbb{P}^{1}-$ естественная проекция, $\mathscr{L}_{X}$ - тавтологический пучок, $Q \subset X$ - гладкий дивизор на $X$, соответствуюший сечению

$$
s_{Q} \in H^{0}\left(X, \mathscr{L}_{X}^{\otimes m} \otimes \pi_{X}^{*} \mathscr{O}_{\mathbb{P}}\left(a_{Q}\right)\right),
$$

$a_{Q} \in \mathbb{Z}_{+}$. Символом $\pi_{Q}: Q \rightarrow \mathbb{P}^{1}$ обозначим проекцию $\left.\pi_{X}\right|_{Q}$. Очевидно, $Q / \mathbb{P}^{1}$ есть гладкое расслоение на гиперповерхности $\Phi$ ано степени $m$ в $\mathbb{P}$. Пусть $W \subset X$ - неприводимая гиперповерхность, соответствуюшая сечению

$$
s_{W} \in H^{0}\left(X, \mathscr{L}_{X}^{\otimes 2 l} \otimes \pi_{X}^{*} \mathscr{O}_{\mathbb{P}^{1}}\left(2 a_{W}\right)\right),
$$

$a_{W} \in \mathbb{Z}_{+}$, причем $W_{Q}=W \cap Q$ - гладкий дивизор на $Q$. Слой $\pi_{Q}^{-1}(t)$ над точкой $t \in \mathbb{P}^{1}$ обозначаем символом $G_{t}$ (или просто $G$, если понятно, о какой точке идет речь, или если это несущественно). Пусть, наконец,

$$
\sigma: V \rightarrow Q
$$

- двойное накрытие, разветвленное над $W_{Q}$. Естественную проекцию на $\mathbb{P}^{1}$ обозначим символом $\pi$, слой $\pi^{-1}(t)$ - символом $F_{t}$ (или просто $F$ ). Легко видеть, что

Pic $V=\mathbb{Z} K_{V} \oplus \mathbb{Z} F$ 
и с точностью до подкрутки на пучок $\mathscr{O}_{\mathbb{P}^{1}}(k), k \in \mathbb{Z}$, пучки $\mathscr{E}$ и $\pi_{*} \mathscr{O}\left(-K_{V}\right)$ на $\mathbb{P}^{1}$ совпадают.

Точнее, пусть $L_{X} \in \operatorname{Pic} X$ - класс тавтологического пучка $\mathscr{L}_{X}, L_{Q}=\left.L_{X}\right|_{Q}-$ его ограничение на $Q$, так что

$$
\operatorname{Pic} Q=\mathbb{Z} L_{Q} \oplus \mathbb{Z} G
$$

Положим $L_{V}=\sigma^{*} L_{Q}$. Легко видеть, что

$$
K_{V}=-L_{V}+\left(a_{1}+\cdots+a_{M}-2+a_{Q}+a_{M}\right) F .
$$

По теореме Лефишеца

$$
A^{2} V=\mathbb{Z} K_{V}^{2} \oplus \mathbb{Z} H_{F}
$$

где $H_{F}=\left(-K_{V} \cdot F\right)$ - класс гиперплоского сечения слоя. Символ $H_{F}$ употребляется в настоящей работе в двух смыслах: в качестве класса коразмерности 2 на $V$ и в качестве гиперплоского сечения слоя, т.е. элемента $A^{1} F$. Каждый раз будет ясно, что именно имеется в виду.

Нетрудно подсчитать, что

$$
\left(K_{V}^{2} \cdot L^{M-1}\right)=2 m\left(4-a_{1}-\cdots-a_{M+1}-a_{Q}-a_{W}\right)+2 a_{Q} .
$$

Поскольку $\left(H_{F} \cdot L^{M-1}\right)=2 m$ и линейная система $\left|L_{V}\right|$ свободна, из неравенства $\left(K_{V}^{2} \cdot L^{M-1}\right) \leqslant 0$ вытекает, что $K_{V}^{2} \notin \operatorname{Int} A_{+}^{2} V$, где $A_{+}^{2} V \subset A^{2} V \otimes \mathbb{R}$ - замкнутый конус эффективных циклов коразмерности 2.

1.3. Условия регулярности вне дивизора ветвления. Пусть $\sigma: F \rightarrow G \subset$ $\mathbb{P}$ - двойная гиперповерхность Фано индекса $1, F \in \mathscr{F}$. Многообразие $F$ реализуется как полное пересечение коразмерности два во взвешенном проективном пространстве

$$
\mathbb{P}(\underbrace{1,1, \ldots, 1}_{M+2}, l)
$$

cм. [7]: $F$ имеет тип $m \cdot 2 l$ и задается парой уравнений

$$
\widetilde{f}\left(x_{0}, \ldots, x_{M+1}\right)=0, \quad u^{2}=\widetilde{g}\left(x_{0}, \ldots, x_{M+1}\right),
$$

где $x_{*}$ - координаты веса $1, u$-координата веса $l, \tilde{f}$ есть уравнение гиперповерхности $G \subset \mathbb{P}=\mathbb{P}(1, \ldots, 1), \widetilde{g}$ есть уравнение гиперповерхности $W \cap \mathbb{P}$.

Пусть $o \in F$ - некоторая точка. Прежде всего, обратим внимание читателя на очевидный факт:

$$
o \neq(\underbrace{0,0, \ldots, 0}_{M+2}, 1) .
$$

Поэтому можно предполагать, что точка $о$ лежит в одной из стандартных аффинных карт $\mathbb{A}^{M+2}$ с координатами

$$
z_{i}=x_{i} / x_{0}, \quad i=1, \ldots, M+1, \quad y=u / x_{0}^{l}
$$


и имеет $z_{*}$-координаты $(0, \ldots, 0)$. Относительно системы координат $\left(z_{*}, y\right)$ аффиннная часть многообразия $F$ задается парой уравнений

$$
f=q_{1}+\cdots+q_{M}=0, \quad y^{2}=g=w_{0}+\cdots+w_{2 l},
$$

где $q_{i}$ и $w_{j}$ суть однородные многочлены от $z_{*}$ степени $i$ или $j$ соответственно. Пусть $p=\sigma(o) \in G$. Точка $p$ лежит на дивизоре ветвления $W$ тогда и только тогда, когда $w_{0}=0$. Если $p \notin W$, то, нормализуя второе уравнение, считаем, что $w_{0}=1$.

Сформулируем сначала условия регулярности вне дивизора ветвления. В этом случае относительно системы аффинных координат $\left(z_{*}, y\right)$ с центром в точке $p=$ $\sigma(o)$ слой $F$ задается уравнениями

$$
\left\{\begin{array}{l}
f=q_{a}+\cdots+q_{m}=0 \\
y^{2}=g=1+w_{1}+\cdots+w_{2 l}
\end{array}\right.
$$

где $a \geqslant 1$.

Положим

$$
\begin{aligned}
\sqrt{g} & =\left(1+w_{1}+\cdots+w_{2 l}\right)^{1 / 2}=1+\sum_{i=1}^{\infty} \gamma_{i}\left(w_{1}+\cdots+w_{2 l}\right)^{i} \\
& =1+\sum_{i=1}^{\infty} \Phi_{i}\left(w_{1}, \ldots, w_{2 l}\right)
\end{aligned}
$$

где $\Phi_{i}\left(w_{1}\left(z_{*}\right), \ldots, w_{2 l}\left(z_{*}\right)\right)$ однородны по $z_{*}$ степени $i \geqslant 1$

$$
\gamma_{i}=(-1)^{i-1} \frac{(2 i-3) ! !}{2^{i} i !}=(-1)^{i-1} \frac{(2 i-3) !}{2^{2 i-2} i !(i-2) !}
$$

- стандартньй коэффициент тейлоровского разложения функции $(1+s)^{1 / 2}$ в точке $s=0$. Очевидно,

$$
\Phi_{i}\left(w_{*}\right)=w_{i}+A_{i}\left(w_{1}, \ldots, w_{i-1}\right)
$$

при $i \leqslant 2 l$. Положим при $i \geqslant 1$

$$
[\sqrt{g}]_{i}=1+\sum_{j=1}^{i} \Phi_{i}\left(w_{*}\right), \quad g^{(i)}=g-[\sqrt{g}]_{i}^{2} .
$$

Легко видеть, что первая ненулевая однородная компонента многочлена $g^{(i)}$ имеет степень $i+1$. Точнее, эта компонента есть в точности

$$
g_{i+1}=2 \Phi_{i+1}\left(w_{1}\left(z_{*}\right), \ldots, w_{i+1}\left(z_{*}\right)\right) .
$$

Условие регулярности в гладкой точке $p \in G$.

(R1.1) Последовательность

$$
q_{1}, \ldots, q_{m}, g_{l+1}, \ldots, g_{2 l-1}
$$

регулярна в $\mathscr{O}_{p, \mathbb{P}} ;$ здесь $a=1$. 
Условие регулярности в двойной точке $p \in G$.

(R1.2) Если $2 l \geqslant m+1$, то система $M-1$ однородных многочленов

$$
q_{2}, \ldots, q_{m}, g_{l+1}, \ldots, g_{2 l-1},
$$

а если $2 l \leqslant m$, то система однородных многочленов

$$
q_{2}, \ldots, q_{m-1}, g_{l+1}, \ldots, g_{2 l}
$$

задает в $\mathbb{P}^{M}=\mathbb{P}\left(T_{p} \mathbb{P}\right)$ кривую, ни одна компонента которой не лежит в гиперплоскости.

Далее, система $M$ однородных уравнений

$$
q_{2}=\cdots=q_{m-1}=g_{l+1}=\cdots=g_{2 l}=0
$$

определяет нульмерную подсхему $Z_{*}$ в $\mathbb{P}^{M}$ такую, что для любой гиперплоскости $P \subset \mathbb{P}^{M}$

$$
\operatorname{deg}\left(P \cap Z_{*}\right)<\lambda_{m, l}=\frac{m !(2 l-1) !}{6(l-1) !}
$$

при $m \geqslant 4$ и

$$
\operatorname{deg}\left(P \cap Z_{*}\right)<\lambda_{3, l}=12 \frac{(2 l-1) !}{(l+1) !}(l-2)
$$

при $m=3$. Если схема $Z_{*}$ приведена, то данное условие означает просто, что никакие $\lambda_{m, l}$ точек не лежат в гиперплоскости.

ЗАМЕчАНИЕ. Поскольку $w_{0}=y(0)=1$, в окрестности особой точки $o \in F$ уравнения

$$
y-[\sqrt{g}]_{i}=0 \quad \text { и } \sigma^{*} g^{(i)}=0
$$

задают один и тот же дивизор. Рассмотрим систему уравнений (3) на слое $F$ (а не на проективизированном касательном пространстве $\mathbb{P}\left(T_{p} \mathbb{P}\right)$ ). Она задает эффективный 1-цикл $C_{*}$ на $F$. По построению его степень есть

$$
\operatorname{deg} C_{*}=2 m ! \frac{(2 l-1) !}{(l-1) !},
$$

а кратность в точке $o \in F$ удовлетворяет оценке

$$
\operatorname{mult}_{o} C_{*} \geqslant m ! \frac{(2 l) !}{l !}=\operatorname{deg} C_{*},
$$

так что в (4) имеет место равенство и $C_{*}$ есть алгебраическая сумма прямых на $F$, т.е. кривых вида $L \ni о$, образ которых $\sigma(L) \subset \mathbb{P}$ есть прямая, причем морфизм $\sigma: L \rightarrow \sigma(L)$ есть изоморфизм. Рассматривая нульмерную схему $Z_{*}$ как эффективный нульмерньй цикл, имеем по построению

$$
Z_{*}=\mathbb{P}\left(T_{o} C_{*}\right) \text {. }
$$

В частности, для любой гиперплоскости $P \subset \mathbb{P}$ одномерная часть схемы

$$
\left\{q_{2}=\cdots=q_{m}=g_{l+1}=\cdots=g_{2 l}=0\right\} \cap \sigma^{-1}(P)
$$

имеет степень не выше $\lambda_{m, l}-1$. Иныпи словами, если все компоненты цикла $C_{*}$ имеют кратность 1 , то не более чем $\lambda_{m, l}-1$ этих прямых содержатся в $\sigma^{-1}(P)$. 
1.4. Условия регулярности на дивизоре ветвления. В этом случае относительно системы аффинных координат $\left(z_{*}, y\right)$ многообразие $F$ задается системой уравнений

$$
\left\{\begin{array}{l}
f=q_{1}+\cdots+q_{m}=0 \\
y^{2}=g=w_{1}+\cdots+w_{2 l}
\end{array} .\right.
$$

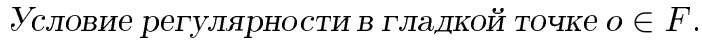

(R2.1) Последовательность однородных многочленов

$$
q_{1}, \ldots, q_{m}
$$

регулярна в $\mathscr{O}_{p, \mathbb{P}}$ и квадратичная форма $q_{2}$ не обрашается тождественно в нуль на плоскости $\left\{q_{1}=w_{1}=0\right\}$.

Отметим, что эта плоскость в силу гладкости точки $o \in F$ имеет коразмерность точно два, т.е. линейные формы $q_{1}$ и $w_{1}$ линейно независимы: плоскость $\left\{q_{1}=\right.$ $\left.w_{1}=0\right\}$ есть касательная плоскость к дивизору ветвления $W \cap G$ морфизма $\sigma_{F}$.

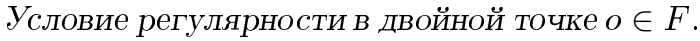

$(\mathrm{R} 2.2)$ Имеем двойное накрытие $\sigma_{F}: F \rightarrow G$, разветвленное над дивизором $W_{G}=W \cap G$. Первое условие -

гладкость гиперповерхности $G$ в точке $p=\sigma(o)$, т.е. $q_{1} \neq 0$.

Далее, дивизор $W_{G}$ имеет в точке $p$ невырожденную квадратичную особенность:

$$
w_{1}=\lambda q_{1},
$$

$\lambda \in \mathbb{C}$. Для удобства обозначений считаем, что $q_{1}=z_{M+1}$. Квадратичный многочлен

$$
\bar{w}_{2}=\left.w_{2}\right|_{\left\{z_{M+1}=0\right\}}
$$

имеет максимальный ранг. Пусть $E_{G} \cong \mathbb{P}^{M-1}$ - исключительный дивизор раздутия $\varphi_{G}: \widetilde{G} \rightarrow G$ точки $p$. Примем $z_{1}, \ldots, z_{M}$ за однородные координаты на $E_{G}$ и положим

$$
W_{E}=\left\{\bar{w}_{2}=0\right\}
$$

- неособая квадратичная гиперповерхность в $E_{G}$. Ограничение однородного многочлена $q_{i}$ на гиперплоскость $q_{M+1}=0$ обозначим символом $\bar{q}_{i}$. Теперь оставшаяся часть условия (R2.2) вьглядит так:

система однородных уравнений

$$
\bar{q}_{2}=\cdots=\bar{q}_{m}=0
$$

задает в $E_{G} \cong \mathbb{P}_{\left(z_{1}: \cdots: z_{M}\right)}^{M-1}$ неприводимое приведенное полное пересечение коразмерности $(m-1)$ - неприводимое подмногообразие $Z_{2 \ldots m}$. Квадрика

$$
\bar{q}_{2}=0
$$

неособа и отлична от $W_{E}$. 
ОПРЕДЕЛЕНИЕ 1.2. Двойная гиперповерхность Фано $F \in \mathscr{F}$ регулярна, если каждая ее гладкая точка регулярна в смысле соответствуюшего условия (R1.1) или (R2.1) и каждая ее особая точка регулярна в смысле соответствующего условия (R1.2) или (R2.2). Обозначение: $F \in \mathscr{F}^{\mathrm{reg}}$.

Условия (R1.1) и (R2.1) совпадают с условиями регулярности работы [7; п. 1.3, определения 1 и 2]. В [7; п. 4.3] было показано, что нерегулярные гладкие двойные пространства образуют во множестве всех гладких двойных гиперповерхностей $\mathscr{F}_{\text {sm }}$ замкнутое подмножество коразмерности не меньше двух. Более того, из вычислений п. 4.3 в [7] следует, что множество двойных гиперповерхностей $Ф$ ано $F$, имеющих хотя бы одну гладкую нерегулярную точку $o \in F$, имеет в $\mathscr{F}$ коразмерность не меньше двух. Поэтому обшая особая двойная гиперповерхность $F \in \mathscr{F} \operatorname{sing}$ имеет в точности одну особую точку, а все ее гладкие точки регулярны. Особая точка $o \in F$ является невырожденной двойной точкой. Если $p=\sigma(o) \notin W_{G}$, то открытость условия (R1.2) показывает, что в окрестности $F \in \mathscr{F}$ имеет место оценка

$$
\operatorname{codim}_{\mathscr{F}_{\text {sing }}}\left(\mathscr{F}_{\text {sing }} \backslash \mathscr{F}_{\text {sing }}^{\text {reg }}\right) \geqslant 1
$$

и потому

$$
\operatorname{codim}_{\mathscr{F}}\left(\mathscr{F}_{\text {sing }} \backslash \mathscr{F}_{\text {sing }}^{\text {reg }}\right) \geqslant 2 .
$$

Если $p=\sigma(o) \in W_{G}$, то открытость условия (R2.2) влечет оценку (5) в окрестности $F \in \mathscr{F}$. Таким образом, оценки (5) и (6) выполнены глобально.

1.5. Начало доказательства теоремы 1. Проверим, что регулярное расслоение $V / \mathbb{P}^{1}$ удовлетворяет условиям (v) и (h). Предположим, что выполнено неравенство

$$
\frac{\text { mult }_{o}}{\operatorname{deg}} Y>\frac{2}{\operatorname{deg} V},
$$

где $o \in F=F_{t}$-гладкая точка, $Y \subset F-$ простой дивизор. Пусть

$$
T=\sigma^{-1}\left(T_{p} G \cap G\right)
$$

- касательный дивизор, $p=\sigma(o)$. В силу условий регулярности $\operatorname{mult}_{o} T=2$. Поскольку $T \subset F$ - гиперплоское сечение, имеем $\operatorname{deg} T=\operatorname{deg} V$, так что

$$
\frac{\text { mult }_{o}}{\operatorname{deg}} T=\frac{2}{\operatorname{deg} V}
$$

и потому $Y \neq T$. Оба подмногообразия $Y, T$ неприводимы, так что пересечение $Y \cap T$ имеет коразмерность 2 относительно $F$ и корректно определен эффективньй цикл $Z=(Y \circ T)$ теоретико-схемного пересечения $Y$ и $T$. Очевидно, цикл $Z$ удовлетворяет неравенству

$$
\frac{\text { mult }_{o}}{\operatorname{deg}} Z>\frac{4}{\operatorname{deg} V} .
$$

Однако в $[7 ;$ п. 3$]$ доказано, что для регулярной точки $o \in F$ это невозможно. Этим справедливость условия (v) доказана. 
Докажем справедливость условия (h). Сначала рассмотрим гладкий случай: $o \in F$ - гладкая точка. Предположим, что неприводимое горизонтальное подмногообразие $Y \subset V$ коразмерности 2 удовлетворяет неравенству

$$
\frac{\text { mult }_{o}}{\operatorname{deg}} Y>\frac{4}{\operatorname{deg} V}
$$

Поскольку $\pi(Y)=\mathbb{P}^{1}$, имеем $Y \neq F$, так что $Z=(Y \circ F)$ - эффективный цикл коразмерности 2 на слое $F$, удовлетворяющий неравенству (7). Как отмечено выше, это невозможно. Условие (h) в гладком случае доказано.

Пусть $o \in F-$ двойная точка. Рассуждая так же, как в гладком случае, построим эффективный цикл $Z=(Y \circ F)$ коразмерности 2 на слое $F$. Поскольку $\frac{\text { mult }_{o}}{\operatorname{deg}} F=2$, цикл $Z$ удовлетворяет неравенству

$$
\frac{\text { mult }_{o}}{\operatorname{deg}} Z>\frac{8}{\operatorname{deg} V}
$$

Покажем, что это невозможно. Без ограничения общности считаем, что $Z \subset F-$ неприводимое подмногообразие коразмерности 2. Его образ на $G$ удовлетворяет оценке

$$
\frac{\operatorname{mult}_{p}}{\operatorname{deg}} \sigma(Z)>\frac{4}{\operatorname{deg} G}
$$

Теперь если $p \in G$ - гладкая точка, то рассуждения работы [11] (работающие без каких-либо изменений для любой степени $\operatorname{deg} G \leqslant \operatorname{dim} G+1)$ показывают, что это невозможно. Если $p \in G$ - двойная точка, то в силу условия (R1.2) однородные многочлены $q_{2}, \ldots, q_{m}$ образуют регулярную последовательность, так что стандартные рассуждения [11] снова дают противоречие (см. [11; п. 3.1]).

Этим справедливость условия (h) доказана полностью.

\section{§2. Особенность слоя вне дивизора ветвления}

\section{1. Гиперкасательные дивизоры и линейные системы. Пусть}

$$
\varphi=\varphi_{F, o}: \widetilde{F} \rightarrow F
$$

- раздутие слоя в произвольной точке $o$,

$$
\varphi_{G}=\varphi_{G, p}: \widetilde{G} \rightarrow G
$$

- раздутие слоя $G$ в точке $p, p=\sigma(o), E=E_{F} \subset \widetilde{F}$ и $E_{G} \subset \widetilde{G}$-исключительные дивизоры. 
ОПРЕДЕЛЕНИЕ 2.1. Линейная система дивизоров

$$
\varphi_{*}\left(\left|k H_{F}-(k+1) E\right|\right)
$$

на $F$ (соответственно линейная система

$$
\left(\varphi_{G}\right)_{*}\left(\left|k H_{G}-(k+1) E_{G}\right|\right)
$$

дивизоров на $G$ ) назьвается $k$-й гиперкасательной линейной системой и обозначается символом $\Lambda_{k}=\Lambda_{k}^{F}$ (соответственно $\left.\Lambda_{k}^{G}\right)$.

Можно сказать, что $\Lambda_{k}$ - это наибольшая линейная подсистема системы $\left|k H_{F}\right|$, собственный прообраз которой удовлетворяет условию

$$
\widetilde{\Lambda}_{k} \subset\left|k H_{F}-(k+1) E\right|
$$

и аналогично для $G$. В общем случае нельзя утверждать, что

$$
\sigma^{*} \Lambda_{k}^{G} \subset \Lambda_{k}
$$

потому, что если $p \in W_{G}$ - неособая точка дивизора ветвления, то двойное накрытие $\sigma: F \rightarrow G$ не продолжается до двойного накрытия $\widetilde{F} \rightarrow \widetilde{G}$ (эти многообразия связаны рациональным отображением степени два, структура которого хотя и проста, но все же это не есть конечный морфизм). Однако если $p \notin W_{G}$ или если $p \in W_{G}$ - двойная точка дивизора ветвления, то включение (8) имеет место.

Символом

$$
\Lambda_{k}^{E}
$$

будем обозначать соответствуюшую линейную систему на исключительном дивизоре:

$$
\Lambda_{k}^{E}=\left.\widetilde{\Lambda}_{k}\right|_{E} \text { или } \Lambda_{k}^{E}=\left.\widetilde{\Lambda}_{k}^{G}\right|_{E_{G}},
$$

в зависимости от контекста. Легко видеть, что

$$
\left(\widetilde{\mathrm{Bs} \Lambda_{k}} \circ E\right)=\operatorname{Bs} \Lambda_{k}^{E}
$$

в теоретико-схемном смысле, в частности, равенство имеет место для соответствуюших эффективных алгебраических циклов, т.е. с учетом кратностей.

Злоупотребляя обозначениями, мы будем называть гиперкасательными линейньми системами некоторые специальные подсистемы гиперкасательных систем, допускаюшие явное описание. Фактически будем пользоваться этими специальньми подсистемами. Пусть $p \in G$ - некоторая точка, $z_{1}, \ldots, z_{M+1}$ - система линейных координат с центром в $p$ и гиперповерхность $G$ задается уравнением

$$
f=q_{a}+q_{a+1}+\cdots+q_{m}=0,
$$

$a=1$ или 2. Тогда

$$
\Lambda_{k}^{G} \supset\left|\sum_{i=a}^{k} s_{k-i} f_{i}\right|,
$$


где

$$
f_{i}=q_{a}+\cdots+q_{i}
$$

$k \geqslant a$ и $s_{j}$ есть произвольный однородный многочлен степени $j$ от переменных $z_{*}$. Включение (9) очевидно, так как

$$
\left.f_{i}\right|_{G}=\left.\left(-q_{i+1}-\cdots-q_{m}\right)\right|_{G}
$$

Предположим теперь, что $p \notin W_{G}$, и построим гиперкасательную систему $\Lambda_{k}$. Очевидно, $\Lambda_{k} \supset \sigma^{*} \Lambda_{k}^{G}$, но система $\Lambda_{k}$ в действительности гораздо больше. Следуя [7], [10], [13], опишем конструкцию гиперкасательных дивизоров, связанных с двойным накрытием $\sigma$. Поскольку $p \notin W_{G}$, можно считать, что гиперповерхность

$$
W_{t}=W \cap \mathbb{P}_{t} \subset \mathbb{P}
$$

задается уравнением

$$
g\left(z_{*}\right)=1+w_{1}+\cdots+w_{2 l}=0
$$

$w_{i}\left(z_{*}\right)$ однородны степени $i$. Записывая формально

$$
\sqrt{g}=1+\sum_{i=1}^{\infty} \Phi_{i}\left(w_{1}, \ldots, w_{2 l}\right)
$$

где $\Phi_{i}\left(w_{1}\left(z_{*}\right), \ldots, w_{2 l}\left(z_{*}\right)\right)$ - однородные многочлены степени $i$ от $z_{*}$, положим для $j \geqslant 1$

$$
[\sqrt{g}]_{j}=1+\sum_{i=1}^{j} \Phi_{i}\left(w_{*}\left(z_{*}\right)\right)
$$

Теперь имеем

$$
\Lambda_{k} \supset\left|\sum_{i=a}^{k} s_{k-i} f_{i}+\sum_{i=l}^{\{k, 2 l-1\}} s_{k-i}^{*}\left(y-[\sqrt{g}]_{i}\right)\right|
$$

где $s_{k-i}^{*}-$ однородные многочлены от $z_{*}$ степени $k-i$; если $k \leqslant l-1$, то правая сумма считается равной нулю. Справедливость включения (11) следует из (9) и следующего факта.

ЛЕмма 2.1. В локальных координатах $z_{*}$ имеем

$$
\left.\left(y-[\sqrt{g}]_{i}\right)\right|_{F}=\left.2 \Phi_{i+1}\left(w_{*}\left(z_{*}\right)\right)\right|_{F}+\cdots
$$

где многоточие обозначает формальный ряд, члены которого - однородные многочлены от переменных $z_{*}$ степени $i+2$ и выше. 
ДоКАЗАТЕЛЬСТво очевидно в силу того, что $\left.\left(y^{2}-g\right)\right|_{F} \equiv 0, g(p)=1$ и имеет место формальное разложение (10).

Отметим, что

$$
\Phi_{i}\left(w_{*}\right)=\frac{1}{2} w_{i}+A_{i}\left(w_{1}, \ldots, w_{i-1}\right) .
$$

Рассмотрим теперь случай, когда $p=\sigma(o) \in W_{G}$. Если дивизор ветвления неособ в точке $p$, то локальное уравнение гиперповерхности $W_{t}$ имеет вид

$$
g\left(z_{*}\right)=w_{1}+\cdots+w_{2 l}=0
$$

где линейные формы $q_{1}, w_{1}$ линейно независимы. Поскольку прообраз дивизора

$$
\left\{\left.w_{1}\right|_{G}=0\right\}
$$

на $F$, очевидно, имеет особенность, получаем

$$
\Lambda_{k} \supset\left|\sum_{i=1}^{k} s_{k-i} f_{i}+s_{k-1} w_{1}\right| .
$$

Если же $p=\sigma(o)$ - особенность дивизора $W_{G}$, то наши методы построения гиперкасательных линейных систем дают лишь включение $\Lambda_{k} \supset \sigma^{*} \Lambda_{k}^{G}$, где $\Lambda_{k}^{G}$ описывается формулой (9).

Условия регулярности позволяют оценить снизу коразмерность базисного множества гиперкасательных систем. Приводимые ниже формулы подразумевают, что отрезок $[a, b] \subset \mathbb{R}$ есть пустое множество, если $b<a$. Для произвольной точки $o \in F$ положим

$$
\begin{gathered}
\mathscr{M}=[a, m-1] \cap \mathbb{Z}_{+}=\{a, \ldots, m-1\}, \\
\text { где } a=\operatorname{mult}_{p} G \in\{1,2\}, p=\sigma(o), \text { и } \\
\mathscr{L}=[l, 2 l+a-3] \cap \mathbb{Z}_{+}=\{l, \ldots, 2 l+a-3\} .
\end{gathered}
$$

Множества $\mathscr{M}, \mathscr{L}$, таким образом, зависят от типа точки $о$. На каждом этапе доказательства точка $о$ предполагается фиксированной, а символы $\mathscr{M}, \mathscr{L}$ обозначают соответствуюшие ей множества.

Для $e=\max \{m-1,2 l-1\}$ гиперкасательную линейную систему $\Lambda_{e}$ будем обозначать символом $\Lambda_{\infty}$.

ПРЕДЛОЖЕНИЕ 2.1. Имеют место следующие оценки.

(i) Если $p=\sigma(o) \notin W_{G}-$ гладкая точка гиперповерхности $G$, то

$$
\operatorname{codim}_{o} \operatorname{Bs} \Lambda_{k} \geqslant \operatorname{codim}_{E} \operatorname{Bs} \Lambda_{k}^{E} \geqslant \#[1, k] \cap \mathscr{M}+\#[1, k] \cap \mathscr{L},
$$

при этом

$$
\operatorname{dim}_{o} \operatorname{Bs} \Lambda_{\infty} \leqslant 1 .
$$

(ii) Если $p=\sigma(o) \notin W_{G}-$ двойная точка гиперповерхности $G$, то $\operatorname{codim} \operatorname{Bs} \Lambda_{k} \geqslant \operatorname{codim}_{E} \operatorname{Bs} \Lambda_{k}^{E} \geqslant \#[2, k] \cap \mathscr{M}+\#[2, k] \cap \mathscr{L}$, 
при этом

$$
\operatorname{Bs} \Lambda_{\infty} \subset C_{*}
$$

(см. п. 1.3). Более того, пусть $P \subset \mathbb{P}, P \ni p,-$ произвольная гиперплоскость, $P_{F}=\sigma^{-1}(P \cap G)$ - соответствующее гиперплоское сечение слоя $F$, $\Lambda_{k}^{P}=\left.\Lambda_{k}\right|_{P_{F}}-$ ограничение линейной системь $\Lambda_{k}$ на $P_{F}$. Тогда при $k \leqslant$ $\max \{m, 2 l\}-2$

$$
\operatorname{codim}_{P_{F}} \operatorname{Bs} \Lambda_{k}^{P} \geqslant \#[2, k] \cap \mathscr{M}+\#[2, k] \cap \mathscr{L},
$$

$a$

$$
\operatorname{dim} \operatorname{Bs} \Lambda_{\infty}^{P} \leqslant 1
$$

причем если в (13) имеет место равенство, то степень одномерной части базисной подсхемы $\operatorname{Bs} \Lambda_{\infty}^{P}$ не превосходит числа $\lambda_{m, l}$.

(iii) Eсли $p=\sigma(o) \in W_{G}-$ гладкая точка на дивизоре ветвления $W_{G}$, то справедливо неравенство

$$
\operatorname{codim}_{o} \operatorname{Bs} \Lambda_{k} \geqslant \operatorname{codim}_{E} \operatorname{Bs} \Lambda_{k}^{E} \geqslant \#[1, k] \cap \mathscr{M}+1 .
$$

(iv) Ecли $p=\sigma(o) \in W_{G}-$ двойная точка на дивизоре ветвления $W_{G}$, то справедливо неравенство

$$
\operatorname{codim}_{o} \operatorname{Bs} \Lambda_{k} \geqslant \operatorname{codim}_{E} \operatorname{Bs} \Lambda_{k}^{E} \geqslant \#[1, k] \cap \mathscr{M} .
$$

ДокАЗАтЕльство. Заменяя гиперкасательные линейные системы $\Lambda_{k}$ построенными выше подсистемами (9), (11) и (12) и применяя условия регулярности (пп. 1.3, 1.4), получаем утверждение предложения.

2.2. Схема доказательства условия (vs). Предположим, что сушествует простой дивизор $Y \subset F=F_{t}$, для которого выполнена оценка

$$
\frac{\operatorname{mult}_{x} \tilde{Y}}{\operatorname{deg} Y}>\frac{1}{m}
$$

где $x \in E$ - бесконечно близкая точка первого порядка, т.е. $E \subset \widetilde{F}$ - исключительный дивизор раздутия точки $o \in F, \varphi: \widetilde{F} \rightarrow F$. Здесь особенность $o \in F$ происходит из особенности гиперповерхности $G=G_{t}$, т.е. $p=\sigma(o) \in G$ - невырожденная двойная точка, $p \notin W$. Положим $\sigma^{-1}(p)=\left\{o, o^{+}\right\}$, и пусть $\varphi_{G}: \widetilde{G} \rightarrow G$ - раздутие точки $p$. Очевидно, $\sigma$ продолжается до морфизма

$$
\widetilde{\sigma}: \widetilde{F} \backslash\left\{o^{+}\right\} \rightarrow \widetilde{G},
$$

причем на исключительном дивизоре $E \subset \widetilde{F}$ морфизм $\widetilde{\sigma}$ есть изоморфизм, что позволяет отождествить $E$ с исключительным дивизором раздутия $\varphi_{G}$ и считать $E$ вложенньг в $\mathbb{T}=\mathbb{P}\left(T_{p} \mathbb{P}\right) \cong \mathbb{P}^{M}$ - в исключительный дивизор раздутия

$$
\varphi_{\mathbb{P}}: \widetilde{\mathbb{P}} \rightarrow \mathbb{P}
$$

точки $p \in \mathbb{P}$. В зависимости от контекста будет подразумеваться одно из вложений $E \subset \widetilde{F}$ или $E \subset \widetilde{G}$.

Покажем, что предположение (14) ведет к противоречию. Для этого применим метод, развитый в [11]. Рассуждения разбиваются на несколько шагов. 


\section{Первый шаг дает}

ПРЕДЛОЖЕНИЕ 2.2. Существует гиперплоскость $P \subset \mathbb{P}, P \ni p$, такая, что $\sigma(Y) \not \subset P$ и эффективный алгебраический иикл $Y_{P}=\left(Y \circ_{F} P_{F}\right)$, где $P_{F}=$ $\sigma^{-1}\left(P_{G}\right), P_{G}=P \cap G$ - гиперплоское сечение, удовлетворяет оценке

$$
\frac{\text { mult }_{o}}{\operatorname{deg}} Y_{P}>\frac{3}{2 m} \text {. }
$$

Символ оF использован для того, чтобы подчеркнуть, что цикл $Y_{P}$ строится в смысле теории пересечений на $F$, а не на $V$. Доказательство предложения см. B $[11]$.

Шаг второй. Рассмотрим многообразие $P_{F} \subset F$. Это неприводимое многообразие размерности $M-1$ с двойной точкой $o \in P_{F}$. Пусть $\varphi_{P}: \widetilde{P} \rightarrow P_{F}-$ раздутие точки $o, E_{P} \subset \widetilde{P}$ - исключительньй дивизор. Очевидно, $\widetilde{P}$ вкладывается в $\widetilde{F}$, а $E_{P}-$ в $E$ как гиперплоское сечение квадрики $E$ относительно вложения $E \hookrightarrow \mathbb{T}$. Поскольку многообразие $\widetilde{F}$ факториально, собственный прообраз $\widetilde{Y}$ есть дивизор Картье. Значит, и эффективный цикл $\widetilde{Y}_{P}=(\widetilde{Y} \circ \widetilde{P})-$ собственный прообраз цикла $Y_{P}$ на $\widetilde{P}-$ есть дивизор Картье,

$$
\widetilde{Y}_{P} \sim a H_{P}-b E_{P}
$$

где $H_{P}-$ класс гиперплоского сечения. В силу предложения 2.2

$$
b>\frac{3}{2} a \text {. }
$$

В силу условия регулярности для касательного дивизора

$$
T=\sigma^{-1}\left(T_{p} G \cap G\right)
$$

имеем mult ${ }_{o} T=6, \operatorname{deg} T=4 m$, так что его собственный прообраз $\widetilde{T} \subset \widetilde{F}$ имеет класс $\widetilde{T} \sim 2 H-3 E$, и потому для его ограничения $\widetilde{T}_{P}=\widetilde{T} \cap \widetilde{P}$ на $\widetilde{P}$ получаем

$$
\widetilde{T}_{P} \sim 2 H_{P}-3 E_{P} .
$$

ПреДЛОЖЕНИЕ 2.3. Пусть $Z \sim \alpha H_{P}-\beta E_{P}-$ эффективный дивизор Картье на $\widetilde{P}$. Предположим, что $\beta>\frac{3}{2} \alpha$. Тогда $Z$ содержит $\widetilde{T}_{P}$ компонентой положительной кратности.

Доказательство дано ниже.

Шаг третий. Запишем эффективньй дивизор $\widetilde{Y}_{P}$ в виде

$$
\widetilde{Y}_{P}=c \widetilde{T}_{P}+Z
$$

где $c \in \mathbb{Z}_{+}$и эффективный дивизор $Z$ не содержит $\widetilde{T}_{P}$ компонентой. Полагая $Z \sim \alpha H_{P}-\beta E_{P}$, получаем из системы уравнений

$$
a=2 c+\alpha, \quad b=3 c+\beta
$$

и условия $2 b>3 a$, что

$$
\beta>\frac{3}{2} \alpha
$$

Согласно предложению 2.3 отсюда вытекает, что $\widetilde{T}_{P}$ - компонента положительной кратности в дивизоре $Z$. Противоречие.

Невозможность оценки (14) и, значит, справедливость условия (vs) для случая особенности $o \in F$, лежащей вне дивизора ветвления, доказаны. 
2.3. Подвижные семейства кривых. Докажем предложение 2.3. Воспользуемся методом работы [11].

Лемма 2.2. Дивизор $T_{P}=T \cap P_{F}$ заметается семейством кривых $\left\{C_{\delta}\right.$, $\delta \in \Delta\}$, общая кривая которого неприводима и удовлетворяет неравенству

$$
\frac{\text { mult }_{o}}{\operatorname{deg}} C_{\delta}>\frac{2}{3}
$$

Прежде всего выведем отсюда предложение 2.3. Пусть $\left\{\widetilde{C}_{\delta}, \delta \in \Delta\right\}$ - собственный прообраз семейства кривых на $\widetilde{P}, \widetilde{T}_{P} \subset \widetilde{P}$ - собственный прообраз дивизора $T_{P}$. Очевидно,

$$
\left(Z \cdot \widetilde{C}_{\delta}\right)=\alpha \operatorname{deg} C_{\delta}-\beta \operatorname{mult}_{o} C_{\delta}<0,
$$

так как $\beta>\frac{3}{2} \alpha$. Следовательно, $\widetilde{C}_{\delta} \subset Z$. Но кривые $\widetilde{C}_{\delta}$ заметают $\widetilde{T}_{P}$, откуда $Z \supset \widetilde{T}_{P}$. Предложение 2.3 доказано.

Доказательство ЛЕммы 2.2. Размерность многообразия $P_{F}$ равна $m+l-2$, размерность дивизора $T_{P} \subset P_{F}$ есть $m+l-3$. Требуемое семейство кривых $\left(C_{\delta}, \delta \in \Delta\right)$ построим, пересекая $T_{P} \mathrm{c} m+l-4$ гиперкасательными дивизорами. Чтобы упорядочить процесс построения, введем новые обозначения:

$$
\begin{aligned}
\mathscr{M} & =\{2, \ldots, m-1\}, \quad \mathscr{L}=\{l, \ldots, 2 l-1\}, \\
c_{e} & =\#[4, e] \cap \mathscr{M}+\#[3, e] \cap \mathscr{L}, \quad e \in \mathbb{Z}_{+} .
\end{aligned}
$$

Здесь и ниже молчаливо предполагается, что отрезок $[a, b] \subset \mathbb{R} \mathrm{c} b<a$ есть пустое множество. При $e \leqslant 2$ имеем $c_{e}=0$, при $e \geqslant \max \{m, 2 l\}-1$ имеем $c_{e}=m+l-4$, если только $l \geqslant 3$. Будем предполагать, что это так и что $m \geqslant 4$. Отметим, что при $m=4$

$$
\#[4, e] \cap \mathscr{M}=0,
$$

так как это множество пусто. Случаи $l=2$ и $m=3$ рассмотрим отдельно. Очевидно,

$$
c_{e+1} \geqslant c_{e} .
$$

Определим упорядочивающую функцию

$$
\chi:\{1, \ldots, m+l-4\} \rightarrow \mathbb{Z}_{+}
$$

формулой

$$
\chi\left(\left[c_{e-1}+1, c_{e}\right] \cap \mathbb{Z}_{+}\right)=e .
$$

Согласно сделанному выше замечанию, если $c_{e-1}=c_{e}$, то формула (17) не содержит никакой информации, так как множество $\left[c_{e-1}+1, c_{e}\right]$ пусто. Отметим, что

$$
c_{e+1}-c_{e} \in\{0,1,2\}
$$

в силу определения (16). Легко проверить, что (17) корректно определяет целочисленную функцию $\chi$. 
Обозначим символом $\Lambda_{i}^{P}$ ограничение гиперкасательной системы $\Lambda_{i}$ на $P_{F}$. Положим

$$
\Lambda^{P}=\prod_{i=1}^{m+l-4} \Lambda_{\chi(i)}^{P} .
$$

Отметим, что в этом произведении гиперкасательная система $\Lambda_{e}$ может появиться не более двух раз, см. (16). Пусть

$$
\mathbb{D}=\left\{D_{i} \in \Lambda_{\chi(i)}^{P}, i=1, \ldots, m+l-4\right\} \in \Lambda^{P}
$$

- общий набор гиперкасательных дивизоров.

ОПРЕДЕЛЕНИЕ 2.2. Семейство замкнутых множеств $\left(\Gamma_{u}, u \in U\right)$ (ко)размерности $i$ на алгебраическом многообразии $Z$ назовем плотным подвижным семейством, если для общего $u \in U$ все неприводимые компоненты множества $\Gamma_{u}$ имеют (ко)размерность $i$ и эти компоненты образуют семейство неприводимых алгебраических подмногообразий, заметаюших $Z$.

ЛЕмма 2.3. При $i=1, \ldots, m+l-4$ замкнутое алгебраическое множество

$$
R_{i}(\mathbb{D})=\bigcap_{j=1}^{i} D_{j} \cap T_{P}
$$

имеет для общего $\mathbb{D} \in \Lambda^{P}$ коразмерность $i$ в $T_{P}$. При $i=1, \ldots, m+l-5$ семейство ииклов

$$
\left(R_{i}(\mathbb{D}), \mathbb{D} \in \Lambda^{P}\right)
$$

есть плотное подвижное семейство циклов коразмерности і на Т.

ДокАЗАТЕЛЬСТво. Положим $R_{0}(\mathbb{D})=T$ и будем рассуждать по индукции по $i=1, \ldots, m+l-4$. Предположим, что утверждение леммы доказано для $i \leqslant j \leqslant m+l-5$ (если $j=0$, то доказьвать нечего). Положим $\chi(j+1)=e$. По определению

$$
R_{j+1}(\mathbb{D})=R_{j}(\mathbb{D}) \cap D_{j+1},
$$

где $D_{j+1} \in \Lambda_{e}^{P}$ - общий дивизор. По определению функции $\chi$ имеем

$$
j+1 \in\left[c_{e-1}+1, c_{e}\right]
$$

Согласно предложению 2.1

$$
\operatorname{codim}_{P_{F}} \operatorname{Bs} \Lambda_{e}^{P} \geqslant c_{e}+1
$$

так что

$$
\left.\operatorname{codim}_{T_{P}} \operatorname{Bs} \Lambda_{e}^{P}\right|_{T_{P}} \geqslant c_{e}
$$

в то время как

$$
\operatorname{codim}_{T_{P}} R_{j}(\mathbb{D})=j \leqslant c_{e}-1
$$


в силу $(16),(17)$. Следовательно, ни одна из неприводимых компонент замкнутого множества $R_{j}(\mathbb{D})$ не содержится в базисном множестве гиперкасательной системы $\Lambda_{e}^{P}$. В частности,

$$
R_{j}(\mathbb{D}) \not \subset D_{j+1}
$$

и потому $R_{j+1}(\mathbb{D})$ - замкнутоемножество, каждая неприводимая компонента которого имеет коразмерность $j+1$ в $T_{P}$, что доказывает первое утверждение леммы. Предположим, что $j \leqslant m+l-6$. Тогда либо

$$
e \leqslant \max \{m, 2 l\}-2,
$$

так что по предложению 2.1 справедлива оценка

$$
\operatorname{codim}_{P_{F}} \operatorname{Bs} \Lambda_{e}^{P} \geqslant c_{e}+2,
$$

усиливаюшая неравенство (18), либо $e=\max \{m, 2 l\}-1$, но тогда $c_{e}=c_{e-1}+2$, поскольку

$$
j+2 \in\left[c_{e-1}+1, c_{e}\right]
$$

так что

$$
\operatorname{codim}_{T_{P}} R_{j}(\mathbb{D})=j=c_{e}-2 .
$$

В любом случае для каждой неприводимой компоненты $Z$ множества $R_{j}(\mathbb{D})$ при $j \leqslant m+l-6$ имеем

$$
\operatorname{codim}_{Z} \operatorname{Bs}\left(\left.\Lambda_{e}^{P}\right|_{Z}\right) \geqslant 2 \text {, }
$$

так что линейная система $\left.\Lambda_{e}^{P}\right|_{Z}$ подвижна. Этим доказано второе утверждение леммы 2.3.

Рассмотрим семейство замкнутых множеств размерности единица

$$
\left(R(\mathbb{D})=R_{m+l-4}(\mathbb{D}), \mathbb{D} \in \Lambda^{P}\right) .
$$

Уже нельзя утверждать, что неприводимые компоненты множества $R(\mathbb{D})$ образуют подвижное семейство кривых: на последнем шаге, т.е. при переходе от поверхностей к кривым, возникают неподвижные компоненты. Однако имеет место разложение

$$
R(\mathbb{D})=\left(T_{P} \circ D_{1} \circ \cdots \circ D_{m+l-4}\right)=\sum_{\delta_{i} \in \Delta} C_{\delta_{i}}+\Phi,
$$

где $\left(C_{\delta}, \delta \in \Delta\right)$ - подвижное семейство кривых, $\Phi$ - некоторый эффективньй 1 -цикл, неподвижная часть семейства кривых $R(\mathbb{D}), \mathbb{D} \in \Lambda^{P}$. Имеем равенство 1-циклов

$$
\Phi=\operatorname{Bs} \Lambda_{\infty}^{P} .
$$

Семейство $\left(C_{\delta}, \delta \in \Delta\right)$ заметает $T_{F}$, eсли оно непусто. Однако по построению

$$
\operatorname{deg} R(\mathbb{D})=4 m \prod_{j=1}^{m+l-4} \chi(j)=4 m\left(\prod_{j=4}^{m-1} j\right)\left(\prod_{j=l}^{2 l-1} j\right)=\frac{2 m !(2 l-1) !}{3(l-1) !},
$$


а в силу условия регулярности

$$
\operatorname{deg} \Phi<\lambda_{m, l}=\frac{m !(2 l-1) !}{6(l-1) !}<\operatorname{deg} R(\mathbb{D})
$$

Следовательно, семейство неприводимых кривых $\left(C_{\delta}, \delta \in \Delta\right)$ непусто и заметает дивизор $T$.

Оценим, наконец, отношение mult $_{o} / \operatorname{deg}$ для общей кривой $C_{\delta}$. Как было отмечено выше, mult $_{o} \Phi=\operatorname{deg} \Phi$ (см. п. 1.3). Кроме того, для общего набора $\mathbb{D} \in \Lambda^{P}$ отношение

$$
\frac{\operatorname{mult}_{o}}{\operatorname{deg}} C_{\delta_{i}}
$$

(в смысле формулы (19)) не зависит от $i$. Следовательно,

$$
\frac{\text { mult }_{o}}{\operatorname{deg}} C_{\delta}=\frac{\operatorname{mult}_{o} R(\mathbb{D})-\operatorname{deg} \Phi}{\operatorname{deg} R(\mathbb{D})-\operatorname{deg} \Phi}
$$

По построению, однако,

$$
\operatorname{mult}_{o} R(\mathbb{D}) \geqslant 6 \prod_{j=1}^{m+l-4}(\chi(j)+1)=6\left(\prod_{j=5}^{m} j\right)\left(\prod_{j=l+1}^{2 l} j\right)=\frac{m !}{4} \cdot \frac{(2 l) !}{l !}
$$

откуда

$$
\frac{\text { mult }_{o}}{\operatorname{deg}} C_{\delta} \geqslant \frac{\frac{m !}{4} \cdot \frac{(2 l) !}{l !}-\lambda_{m, l}}{\frac{2 m !}{3} \cdot \frac{(2 l-1) !}{(l-1) !}-\lambda_{m, l}}>\frac{2}{3}
$$

в силу выбора числа $\lambda_{m, l}$. Этим лемма доказана для $m \geqslant 5, l \geqslant 3$.

Если $l=2$, то проходят приведенные рассуждения с единственной модификацией: вместо (16) используем формулу

$$
c_{e}=\#[3, e] \cap \mathscr{M}+\#[3, e] \cap \mathscr{L},
$$

$e \in \mathbb{Z}_{+}$. В этом случае в линейную систему $\Lambda_{2}^{P}$ добавляется независимый гиперкасательньй дивизор и коразмерность ее базисного множества (и всех последующих гиперкасательных систем $\Lambda_{j}^{P}, j \geqslant 3$ ) повьшшается на единищу по сравнению с уже рассмотренньм случаем $l \geqslant 3$. Это и позволяет изменить определение числа $c_{e}$ и, соответственно, сдвинуть на единицу функцию $\chi$. Остальные рассуждения полностью аналогичны разобранному выше случаю $l \geqslant 3$.

В случае $m \geqslant 3$ положение несколько сложнее. Чтобы получить необходимую коразмерность базисного множества гиперкасательной системы, необходимо использовать набор гиперкасательных дивизоров

$$
\mathbb{D}=\left\{D_{i} \in \Lambda_{l+i}^{P} \mid i=1, \ldots, l-1\right\}
$$


Обратим внимание читателя, что первый дивизор в этом наборе взят в линейной системе $\Lambda_{l+1}^{P}$, т.е. в отличие от разобранного вьшше случая $m \geqslant 4$ система $\Lambda_{l}^{P}$ пропушена. В результате вновь получается подвижное семейство замкнутых алгебраических множеств

$$
R_{k}(\mathbb{D})=\left(\bigcap_{j=1}^{k} D_{j}\right) \cap T
$$

при $k \leqslant 2 l-2$, причем неприводимые компоненты множеств $R_{k}(\mathbb{D})$ образуют семейство и заметают $T$. В семействе кривых $R_{2 l-1}(\mathbb{D})$ снова выбрасьваем неподвижную часть $\Phi$ степени $\operatorname{deg} \Phi<\lambda_{3, l}=12(l-2) \frac{(2 l-1) !}{(l+1) !}$ и получаем семейство неприводимых кривых $\left(C_{\delta}, \delta \in \Delta\right)$, заметающих $T$ и удовлетворяющих оценке (15). Лемма 2.2 доказана полностью.

\section{§3. Особенность слоя на дивизоре ветвления}

3.1. Обозначения и анализ условия регулярности. Имеем двойное накрытие

$$
F=F_{t} \stackrel{\sigma}{\longrightarrow} G=G_{t} \subset \mathbb{P}=\mathbb{P}^{M+1},
$$

$G \subset \mathbb{P}$ - гладкая гиперповерхность степени $m \leqslant M-1$. В точке $p \in G$ гиперповерхность ветвления $W_{G}=W \cap G$ имеет изолированную квадратичную особенность, так что $o=\sigma^{-1}(p) \in F$ есть (изолированная) невырожденная двойная точка слоя $F$. Имеем коммутативную диаграмму отображений

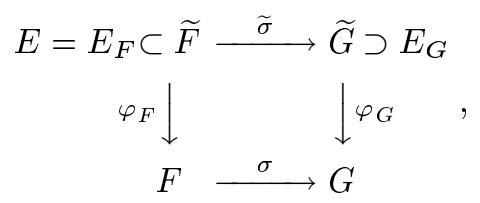

где $\varphi_{F}$ и $\varphi_{G}$ - раздутия точек $o \in F$ и $p \in G$ соответственно, $E_{F}$ и $E_{G}-$ исключительные дивизоры, $\widetilde{\sigma}$ - двойное накрытие, разветвленное над $\widetilde{W}_{G} \subset \widetilde{G}-$ собственным прообразом дивизора $W_{G}$. При этом

$$
\widetilde{\sigma}_{E}=\left.\widetilde{\sigma}\right|_{E}: E \rightarrow E_{G} \cong \mathbb{P}^{M-1}
$$

- двойное накрытие, разветвленное над квадрикой

$$
W_{E}=\widetilde{W}_{G} \cap E_{G}
$$

Символом $H_{E}$ обозначаем гиперплоское сечение квадрики $E$ относительно стандартного вложения $E \hookrightarrow \mathbb{P}^{M}$, Pic $E=\mathbb{Z} H_{E}$.

Пусть $W_{t}=W \cap \mathbb{P}_{t}$ задается уравнением

$$
h=w_{1}+w_{2}+\cdots+w_{2 l}=0,
$$

а $G$ - уравнением

$$
f=q_{1}+q_{2}+\cdots+q_{m}=0
$$


относительно аффинных координат $z_{*}=\left(z_{1}, \ldots, z_{M+1}\right)$ с центром в точке $p$. Дивизор $W_{G}$ имеет в $p$ невырожденную квадратичную особенность, так что $w_{1}=\lambda q_{1}$, для простоты обозначений считаем, что $q_{1}=z_{M+1}$. Квадратичный многочлен $\bar{w}_{2}=\left.w_{2}\right|_{\left\{z_{M+1}=0\right\}}$ имеет максимальный ранг. Принимаем $z_{1}, \ldots, z_{M}$ за однородные координаты на $E_{G}$, тогда

$$
\widetilde{\sigma}_{E}: E \rightarrow E_{G} \cong \mathbb{P}^{M-1}
$$

разветвлено над неособой квадрикой $W_{E}=\left\{\bar{w}_{2}=0\right\}$. Для произвольной точки $y \in E_{G} \backslash W_{E}$ пусть $C(y) \subset E_{G}-$ квадратичный конус, состояший из всех прямых $L \subset E_{G}$, содержаших $y$ и касаюшихся $W_{E}$. Более формально, пусть

$$
\pi_{y}: E_{G} \backslash\{y\} \rightarrow \mathbb{P}^{M-2}
$$

- проекция из точки $y$. Ее ограничение на квадрику $W_{E}$

$$
\left.\pi_{y}\right|_{W_{E}}: W_{E} \rightarrow \mathbb{P}^{M-2}
$$

есть двулистное накрытие, разветвленное над квадрикой $Q(y) \subset \mathbb{P}^{M-2}$. Теперь

$$
C(y)=\overline{\pi_{y}^{-1}(Q(y))} \text {. }
$$

Очевидно, $C(y)$ - квадратичный конус с вершиной в точке $y$. Поскольку квадрика $W_{E}$ неособа, конус $C(y)$ имеет $y$ единственной особой точкой.

Ограничение однородного многочлена $q_{i}$ на гиперплоскость $q_{M+1}=0$ обозначаем символом $\bar{q}_{i}$. Согласно условию регулярности система однородных уравнений

$$
\bar{q}_{2}=\cdots=\bar{q}_{m}=0
$$

задает в $E_{G}$ неприводимое приведенное полное пересечение коразмерности $(m-1)$ - неприводимое подмногообразие $Z_{2 \ldots m}$, причем квадрика $\bar{q}_{2}=0$ неособа и отлична от $W_{E}$.

Лемма 3.1. Если выполнено условие (R2.2), то подмногообразие $Z_{2 . . m}$ не содержится ни в каком квадратичном конусе $C(y), y \in E_{G} \backslash W_{E}$, и ни в какой касательной гиперплоскости $T_{y} W_{E}, y \in W_{E}$.

ДокАЗАТЕЛЬСТво. Положим

$$
Z_{2 \ldots j}=\left\{z \in \mathbb{P}^{M-1} \mid \bar{q}_{2}=\cdots=\bar{q}_{j}=0\right\} .
$$

Легко видеть, что $Z_{2 \ldots j}$ есть неприводимое приведенное полное пересечение коразмерности $j$. Из длинной точной последовательности когомологий получаем

$$
h^{0}\left(\mathscr{O}_{Z_{2}}(2)\right)=\cdots=h^{0}\left(\mathscr{O}_{Z_{2 \ldots j}}(2)\right)=\cdots=h^{0}\left(\mathscr{O}_{Z_{2 \ldots m}}(2)\right),
$$

причем отображение ограничения

$$
H^{0}\left(\mathscr{O}_{\mathbb{P} M-1}(2)\right) \rightarrow H^{0}\left(\mathscr{O}_{Z_{2 \ldots m}}(2)\right)
$$


сюръективно, откуда следует, что $Z_{2} \ldots m$ содержится в единственной квадрике $Z_{2}$ и потому не содержится ни в каком квадратичном конусе $C(y), y \in E_{G} \backslash W_{E}$. Аналогичным образом, отображение ограничения

$$
H^{0}\left(\mathscr{O}_{\mathbb{P}^{M-1}}(1)\right) \rightarrow H^{0}\left(\mathscr{O}_{Z_{2 \ldots m}}(1)\right)
$$

есть изоморфизм, так что $Z_{2} \ldots m$ не содержится ни в какой гиперплоскости, в частности ни в какой гиперплоскости вида $T_{y} W_{E}, y \in W_{E}$.

Зафиксируем простой дивизор $R \subset F$, и пусть $\widetilde{R} \subset \widetilde{F}$ - его собственный прообраз. Зафиксируем также произвольную точку $x \in E$, лежащую вне дивизора ветвления накрытия $\widetilde{\sigma}_{E}$, т.е. $\widetilde{\sigma}(x) \notin W_{E}$.

ПРЕДЛОЖЕНИЕ 3.1. Имеет место оценка

$$
\mu=\operatorname{mult}_{x} \widetilde{R} \leqslant \frac{1}{m} \operatorname{deg} R .
$$

ЗАмечаниЕ. Для некоторого $k \geqslant 1$ имеем $R \sim k H_{F}$, где $H_{F}=\sigma^{*} H_{G}-$ гиперплоское сечение. Поскольку, очевидно, $\operatorname{deg} R=2 m k$, оценка (20) принимает вид неравенства

$$
\mu \leqslant 2 k
$$

3.2. Начало доказательства условия (vs). Предположим противное: $\mu>2 k$. Имеем представление

$$
\widetilde{R} \sim k \varphi_{F}^{*} H_{F}-\nu E
$$

причем mult $_{o} R=2 \nu$.

Лемма 3.2. Имеет место оценка $\nu \leqslant 2 k$.

ДокАЗАТЕЛьСтво. Предположим противное: $\nu>2 k$. Тогда

$$
\frac{\text { mult }_{o}}{\operatorname{deg}} R>\frac{2}{m} .
$$

Положим $\bar{R}=\sigma(R) \subset G$. Это простой дивизор на гладкой гиперповерхности $G \subset \mathbb{P}$. Поскольку $\sigma: R \rightarrow \bar{R}$ - конечный морфизм, имеем неравенство

$$
\frac{\operatorname{mult}_{p}}{\operatorname{deg}} \bar{R}>\frac{2}{m} \text {. }
$$

Однако этого не может быть, потому что $p \in G$ - регулярная точка. В самом деле, касательньй дивизор $T_{1}^{+}=T_{p} G \cap G$ удовлетворяет равенству

$$
\frac{\operatorname{mult}_{p}}{\operatorname{deg}} T_{1}^{+}=\frac{2}{m},
$$

так что $\bar{R} \neq T_{1}^{+}$и $\left(\bar{R} \circ T_{1}^{+}\right)$- эффективный цикл коразмерности $2\left(T_{1}^{+}\right.$очевидньм образом неприводим). 
Поскольку mult ${ }_{p} T_{1}^{+}=2$, имеем неравенство

$$
\operatorname{mult}_{p}\left(\bar{R} \circ T_{1}^{+}\right) \geqslant 2 \operatorname{mult}_{p} \bar{R} .
$$

Учитывая, что $\operatorname{deg}\left(\bar{R} \circ T_{1}^{+}\right)=\operatorname{deg} \bar{R}$, получаем, что существует неприводимая компонента $Y_{2}$ цикла $\left(\bar{R} \circ T_{1}^{+}\right)$, удовлетворяюшая оценке

$$
\frac{\operatorname{mult}_{p}}{\operatorname{deg}} Y_{2} \geqslant 2 \frac{\operatorname{mult}_{p}}{\operatorname{deg}} \bar{R}
$$

Пусть, как обычно, $f=q_{1}+q_{2}+\cdots+q_{m}$ - многочлен, задающий гиперповерхность $G$ относительно системы координат $z_{*}$ с началом в точке $p$. Полагая

$$
f=q_{1}+q_{2}+\cdots+q_{i},
$$

построим гиперкасательные системы

$$
\Lambda_{i}^{G}=\left|\sum_{j=1}^{i} f_{j} s_{i-j}\right|_{G}=0 \mid
$$

и рассмотрим стандартные гиперкасательные дивизоры

$$
T_{i}^{+}=\left\{\left.f_{i}\right|_{G}=0\right\} \in \Lambda_{i}^{G} .
$$

Положим

$$
T_{i}=\sigma^{*} T_{i}^{+}, \quad \Lambda_{i}=\sigma^{*} \Lambda_{i}^{G} .
$$

Эти дивизоры и линейные системы будут играть основную роль ниже. Пока отметим лишь, что в силу условия регулярности имеем

$$
\operatorname{codim}_{G} \operatorname{Bs} \Lambda_{i}^{G}=i
$$

(на самом деле $\operatorname{Bs} \Lambda_{i}^{G}=T_{1}^{+} \cap \cdots \cap T_{i}^{+}$). Пусть

$$
\mathbb{D}=\left(D_{1}, \ldots, D_{m-1}\right) \in \prod_{j=1}^{m-1} \Lambda_{i}^{G}
$$

- общий набор дивизоров. Построим по индукции последовательность неприводимых подмногообразий $Y_{i}, i=1, \ldots, m-1$, удовлетворяюшую следующим свойствам:

(i) $Y_{1}=\bar{R}, Y_{2}$ построено выше, $\operatorname{codim}_{G} Y_{i}=i$;

(ii) $Y_{i+1} \subset Y_{i}, Y_{i} \not \subset D_{i+1}, Y_{i+1}$ - неприводимая компонента замкнутого множества $Y_{i} \cap D_{i+1}$;

(iii) имеет место неравенство

$$
\frac{\operatorname{mult}_{p}}{\operatorname{deg}} Y_{i+1} \geqslant \frac{i+2}{i+1} \cdot \frac{\operatorname{mult}_{p}}{\operatorname{deg}} Y_{i} .
$$


Такое построение возможно, потому что

$$
\operatorname{codim}_{G} \Lambda_{i+1}^{G}=i+1>\operatorname{codim}_{G} Y_{i}
$$

так что для обшего дивизора $D_{i+1} \subset \Lambda_{i+1}^{G}$ имеем $Y_{i} \not \subset D_{i+1}$. Вьполнения свойства (iii) можно добиться, потому что $\Lambda_{j}^{G} \subset\left|j H_{G}\right|$ и $\operatorname{mult}_{p} \Lambda_{j}^{G}=j+1$.

Теперь для неприводимого подмногообразия $Y=Y_{m-1}$ имеем оценку:

$$
1 \geqslant \frac{\operatorname{mult}_{p}}{\operatorname{deg}} Y \geqslant \underbrace{\frac{m}{m-1} \cdot \frac{m-1}{m-2} \cdots \frac{4}{3} \cdot \frac{2}{1}}_{\substack{\| \\ 2 m / 3}} \cdot \frac{\operatorname{mult}_{p}}{\operatorname{deg}} \bar{R},
$$

откуда получаем

$$
\frac{\operatorname{mult}_{p}}{\operatorname{deg}} \bar{R} \leqslant \frac{3}{2 m} .
$$

Таким образом, максимум отношения mult $_{p} / \mathrm{deg}$ достигается на касательном дивизоре $T_{p} G \cap G$ и равен двум. Противоречие. Лемма доказана.

3.3. Гиперкасательные дивизоры и касательные конусы. Итак, $\nu \leqslant$ $2 k<\mu$. С другой стороны,

$$
\mu=\operatorname{mult}_{x} \widetilde{R} \leqslant \operatorname{mult}_{x}(\widetilde{R} \circ E) \leqslant \operatorname{deg}(\widetilde{R} \circ E)=2 \nu .
$$

Положим $B=T_{x} E \cap E$, где квадрика $E$ считается стандартно вложенной в $\mathbb{P}^{M}$. Согласно [11; п. 3.5, лемма 5$]$

$$
\operatorname{mult}_{B} \widetilde{R} \geqslant \frac{1}{2}(\mu-\nu)
$$

а для эффективного цикла $R_{E}=(\widetilde{R} \circ E)$ имеем

$$
\operatorname{mult}_{B} R_{E} \geqslant \mu-\nu
$$

Пусть $\widetilde{T}_{i}, \widetilde{\Lambda}_{i}-$ собственные прообразы дивизоров $T_{i}$ и линейных систем $\Lambda_{i}$ на раздутии $\widetilde{F}$ слоя $F$. Легко видеть, что $\widetilde{T}_{i} \in \widetilde{\Lambda}_{i}$, поскольку по построению

$$
\operatorname{mult}_{o} \Lambda_{i}=\operatorname{mult}_{o} T_{i}
$$

Пусть также

$$
\mathbb{T}_{i}=\left(\widetilde{T}_{i} \circ E\right)=\widetilde{T}_{i} \cap E
$$

- проективизированный касательньй конус к дивизору $T_{i}$ в точке $o$. Напомним, что квадрика $E$ реализуется как двойное накрытие $\widetilde{\sigma}_{E}: E \rightarrow E_{G} \cong \mathbb{P}^{M-1}$, разветвленное над квадрикой $W_{E}$. Если $\left(z_{1}, \ldots, z_{M+1}\right)$ - система аффинных координат на $\mathbb{P}$ с центром в точке $p$, то, считая, что $q_{1} \equiv z_{M+1}$, можно рассматривать $\left(z_{1}, \ldots, z_{M}\right)$ как однородные координаты на проективном пространстве $E_{G}$. В терминах этих координат гиперповерхность $\mathbb{T}_{i} \subset E$ задается уравнением

$$
\left.\left(\widetilde{\sigma}_{E}\right)^{*} q_{i+1}\right|_{E_{G}}
$$


Пусть, наконец,

$$
\Lambda_{i}^{E}=\left.\widetilde{\Lambda}_{i}\right|_{E}
$$

- проективизированная касательная система линейной системы $\Lambda_{i}$ в точке $o$. Уравнения дивизоров этой системы получаются $\widetilde{\sigma}_{E}$-подъемом на $E$ уравнений

$$
\sum_{j=1}^{i} \bar{q}_{j+1} \bar{s}_{i-j},
$$

где \# означает ограничение многочлена \# на гиперплоскость $z_{M+1}=0$. Очевидньмм образом

$$
\mathbb{T}_{i} \sim(i+1) H_{E}, \quad \Lambda_{i}^{E} \subset\left|(i+1) H_{E}\right|,
$$

кроме того, из уравнений (22) имеем непосредственно

$$
\operatorname{Bs~} \Lambda_{i}=T_{1} \cap \cdots \cap T_{i}, \quad \operatorname{Bs} \Lambda_{i}^{E}=\mathbb{T}_{1} \cap \cdots \cap \mathbb{T}_{i}
$$

оба равенства в теоретико-схемном смысле.

3.4. Построение новых циклов. В силу условия регулярности множество $\mathbb{T}_{1} \cap \cdots \cap \mathbb{T}_{i}$ неприводимо и не содержится в дивизоре $B$ для всех $i=1, \ldots, m-1$. Пусть

$$
\mathscr{L}=\left(L_{2}, \ldots, L_{m-1}\right) \in \Lambda_{2} \times \cdots \times \Lambda_{m-1}
$$

- общий набор гиперкасательных дивизоров. Собственный прообраз гиперкасательного дивизора $L_{j}$ на $\widetilde{F}$ обозначаем символом $\widetilde{L}_{j}$, а его проективизированный касательный конус в точке $o \in F-$ символом

$$
\mathbb{L}_{j}=\left(\widetilde{L}_{j} \circ E\right)
$$

Для общего дивизора $L_{j} \in \Lambda_{j}$ имеем $\mathbb{L}_{j}=\widetilde{L}_{j} \cap E$.

Лемма 3.3. (i) Пусть $Y \subset F$ - фиксированное неприводимое подмногообразие коразмерности $l \leqslant m-2$. Для общего дивизора $L_{l+1} \in \Lambda_{l+1}$ имеем $Y \not \subset L_{l+1}$.

(ii) Пусть $Y \subset E$ - фиксированное неприводимое подмногообразие коразмерности $l \leqslant m-2$. Для общего дивизора $L_{l+1} \in \Lambda_{l+1}$ имеем $Y \not \subset \mathbb{L}_{l+1}$.

ДОКАЗАТЕЛЬСТВо. В силУ условия регулярности

$$
\operatorname{codim}_{F} \operatorname{Bs} \Lambda_{l+1}=l+1, \quad \operatorname{codim}_{E} \operatorname{Bs} \Lambda_{l+1}^{E}=l+1
$$

и для обшего дивизора $L_{j} \in \Lambda_{j}$ имеем $\mathbb{L}_{j} \in \Lambda_{j}^{E}$. Лемма доказана. 
СлЕДСТВИЕ 3.1. Для общего набора $\mathscr{L}$ имеем

$$
\begin{aligned}
\operatorname{codim}_{F}\left(R \cap L_{2} \cap \cdots \cap L_{m-1}\right) & =m-1, \\
\operatorname{codim}_{E}\left(R_{E} \cap \mathbb{L}_{2} \cap \cdots \cap \mathbb{L}_{m-1}\right) & =m-1 .
\end{aligned}
$$

Отсюда получаем, что корректно определены эффективные алгебраические циклы коразмерности $m-1$ на $F$ и $E$ соответственно:

$$
R^{+}=\left(R \circ L_{2} \circ \cdots \circ L_{m-1}\right)
$$

и

$$
R_{E}^{+}=\left(R_{E} \circ \mathbb{L}_{2} \circ \cdots \circ \mathbb{L}_{m-1}\right),
$$

причем (для общего набора $\mathscr{L}$ )

$$
R_{E}^{+}=\left(\widetilde{R}^{+} \circ E\right)
$$

есть проективизированный касательный конус к циклу $R^{+}$в точке $o$. Опишем строение этих эффективных циклов. Прежде всего, имеем

$$
\begin{aligned}
\operatorname{deg} R^{+} & =2 k m \cdot(m-1) !=2 k m !, \\
\operatorname{mult}_{o} R^{+} & =\operatorname{deg} R_{E}^{+}=2 \nu \cdot 3 \cdots m=\nu m ! .
\end{aligned}
$$

ЛЕмма 3.4. Пусть $Y$ - неприводимая компонента иикла $R^{+}$. Если $Y \subset T_{1}$, mo

$$
Y=T_{1} \cap T_{2} \cap \cdots \cap T_{m-1} .
$$

ДокАЗАТЕЛЬСТво. По построению уравнение дивизора $L_{i}$ имеет вид

$$
f_{1} s_{i-1}+f_{2} s_{i-2}+\cdots+f_{i} s_{0},
$$

где $s_{j}$ - однородный многочлен степени $j$ от координат $z_{*}$. Поскольку гиперкасательные дивизоры $L_{i}$ предполагаются общими, можно считать, что $s_{0} \neq 0$ и потому нормализовать уравнение условием $s_{0}=1$. Предположим, что $Y \subset T_{1}$. Тогда на $Y$ обрашаются в нуль следующие многочлены:

$$
\begin{aligned}
& f_{1}, \\
& f_{1} s_{2,1}+f_{2}, \\
& f_{1} s_{3,2}+f_{2} s_{3,1}+f_{3}, \\
& \ldots \ldots \ldots \ldots \ldots \ldots \ldots \ldots \ldots \ldots \ldots \ldots \ldots \ldots \ldots \ldots \ldots \ldots \ldots \\
& f_{1} s_{m-1, m-2}+\cdots+f_{m-2} s_{m-1,1}+f_{m-1},
\end{aligned}
$$

где $s_{i, j}$ - однородный многочлен степени $j$. Поэтому

$$
\left.\left.\left.f_{1}\right|_{Y} \equiv f_{2}\right|_{Y} \equiv \cdots \equiv f_{m-1}\right|_{Y} \equiv 0,
$$

так что $Y \subset T_{1} \cap T_{2} \cap \cdots \cap T_{m-1}$, но последнее множество неприводимо и имеет ту же размерность, что и $Y$. Лемма 3.4 доказана. 


\section{5. Степени и кратности. Положим}

$$
T=T_{1} \cap T_{2} \cap \cdots \cap T_{m-1}, \quad \mathbb{T}=\mathbb{T}_{1} \cap \mathbb{T}_{2} \cap \cdots \cap \mathbb{T}_{m-1} .
$$

Учитывая, что $T=\left(T_{1} \circ \cdots \circ T_{m-1}\right)$ и $\mathbb{T}=\left(\mathbb{T}_{1} \circ \cdots \circ \mathbb{T}_{m-1}\right)_{E}$, нетрудно убедиться, что

$$
\operatorname{deg} T=\operatorname{mult}_{o} T=\operatorname{deg} \mathbb{T}=2 m !
$$

Запишем теперь

$$
R^{+}=a T+R^{\#}, \quad R_{E}^{+}=a \mathbb{T}+R_{E}^{\#},
$$

где $a \in \mathbb{Z}_{+}$, эффективный цикл $R^{\#}$ однозначно определен требованием, чтобы подмногообразие $T$ не было его компонентой, и

$$
R_{E}^{\#}=\left(\widetilde{R}^{\#} \circ E\right)
$$

- проективизированный касательный конус к $R^{\#}$ в точке $o$. Отметим, что неприводимое подмногообразие $\mathbb{T}$, вообше говоря, может быть компонентой эффективного цикла $R_{E}^{\#}$.

Лемма 3.5. Имеет место оценка

$$
2 \text { mult }_{o} R^{\#} \leqslant \operatorname{deg} R^{\#} .
$$

ДокАЗАТЕЛЬСтво. Пусть $Y$ - неприводимая компонента цикла $R^{\#}$. По построению $Y \neq T$; следовательно, по лемме $3.4 Y \not \subset T_{1}$. Поэтому замкнутое множество

$$
T_{1} \cap \operatorname{Supp} R^{\#}
$$

имеет коразмерность $m$, так что корректно определен эффективный цикл

$$
R^{*}=\left(R^{\#} \circ T_{1}\right) .
$$

Теперь имеем стандартную цепочку оценок

$$
2 \text { mult }_{o} R^{\#} \leqslant \operatorname{mult}_{o} R^{*} \leqslant \operatorname{deg} R^{*}=\operatorname{deg} R^{\#},
$$

что и требовалось доказать.

Как и в следствии 3.1 , из леммы 3.5 получаем, что множество

$$
B \cap \mathbb{L}_{2} \cap \cdots \cap \mathbb{L}_{m-1}
$$

имеет в $E$ коразмерность $m-1$. Пусть $B^{+}$- часть эффективного равноразмерного цикла $R_{E}^{+}$, носитель которой содержится в $B$ :

$$
R_{E}^{+}=\sum_{i \in I} r_{i} Y_{i}, \quad B^{+}=\sum_{i \in I, Y_{i} \subset B} r_{i} Y_{i} .
$$


ЛЕмма 3.6. Имеет место оценка

$$
\operatorname{deg} B^{+} \geqslant(\mu-\nu) m !
$$

ДокАЗАТЕЛЬство. В самом деле, в силу (21) имеем

$$
R_{E}=(\mu-\nu) B+\Delta
$$

где $\Delta$ - эффективный цикл. Далее,

$$
\operatorname{deg}\left(B \circ \mathbb{L}_{2} \circ \cdots \circ \mathbb{L}_{m-1}\right)=2 \cdot 3 \cdots m=m !,
$$

что и доказывает лемму.

Лемма 3.7. Пусть $Y \subset \mathbb{L}_{2} \cap \cdots \cap \mathbb{L}_{m-1}$ - неприводимое подмногообразие коразмерности $m-1$ в Е. Если $Y \subset \mathbb{T}_{1}$, mо $Y=\mathbb{T}$.

ДоказАТЕльство. Уравнение дивизора $\mathbb{L}_{i}$ в однородных координатах $z_{*}$ имеет вид

$$
q_{2} s_{i-1}+\cdots+q_{i+1},
$$

где $s_{j}$ - однородный многочлен степени $j$. Если $Y \subset \mathbb{T}_{1}$, то на $Y$ обрашаются в нуль многочлены

$$
\begin{aligned}
& q_{2}, \\
& q_{2} s_{2,1}+q_{3}, \\
& \ldots \ldots \ldots \ldots \ldots \ldots \ldots \ldots \ldots \ldots \ldots \ldots \ldots \ldots \ldots \ldots \ldots \\
& q_{2} s_{m-1, m-2}+\cdots+q_{m-1} s_{m-1,1}+q_{m},
\end{aligned}
$$

где $\operatorname{deg} s_{i, j}=j$. Следовательно,

$$
\left.\left.\left.q_{2}\right|_{Y} \equiv q_{3}\right|_{Y} \equiv \cdots \equiv q_{m}\right|_{Y} \equiv 0
$$

т.е. $Y \subset \mathbb{T}$, а ввиду совпадения размерностей $Y=\mathbb{T}$. Лемма доказана.

СлЕДСТВИЕ 3.2. Ни одна компонента замкнутого множества

$$
B \cap \mathbb{L}_{2} \cap \cdots \cap \mathbb{L}_{m-1}
$$

не содержится в $\mathbb{T}_{1}$.

ДокАЗАТЕЛЬСтво. Пусть $Y$ - такая компонента и $Y \subset \mathbb{T}_{1}$. Согласно предыдущей лемме $Y=\mathbb{T}$. Поэтому $\mathbb{T} \subset B$ : противоречие с условием регулярности. Следствие доказано. 
Завершим, наконец, доказательство предложения 3.1. Из представлений (23) имеем

$$
\begin{aligned}
\operatorname{deg} R^{+} & =2 k m !=2 a m !+\operatorname{deg} R^{\#}, \\
\operatorname{mult}_{o} R^{+} & =\nu m !=2 a m !+\operatorname{mult}_{o} R^{\#} .
\end{aligned}
$$

В силу следствия 3.2 эффективньй цикл $B^{+}$целиком содержится в $R_{E}^{\#}$. В частности,

$$
\operatorname{deg} R_{E}^{\#} \geqslant \operatorname{deg} B^{+} \geqslant(\mu-\nu) m ! .
$$

Ho $\operatorname{deg} R_{E}^{\#}=$ mult $_{o} R^{\#}$. Применяя лемму 3.5 , получаем

$$
2(\nu m !-2 a m !) \leqslant 2 k m !-2 a m ! .
$$

Неравенство (24) перепишем в виде

$$
\nu m !-2 a m ! \geqslant(\mu-\nu) m ! .
$$

После несложных преобразований получаем пару неравенств

$$
\begin{array}{r}
k+a \geqslant \nu, \\
2 \nu-2 a \geqslant \mu,
\end{array}
$$

откуда очевидным образом вытекает искомая оценка (20).

Однако мы предполагали, что $\mu>2 k$. Полученное противоречие завершает доказательство предложения 3.1.

Этим теорема 1 доказана.

\section{Список литературы}

1. Graber T., Harris J., Starr J. Families of rationally connected varieties // J. Amer. Math. Soc. 2002. V. 16. № 1. P. 57-67.

2. Brown G., Corti A., Zucconi F. Birational geometry of 3-fold Mori fibre spaces // Preprint, 2003; arXiv: math.AG/0307301.

3. Пухликов $A$. В. Бирациональные автоморфизмы трехмерных алгебраических многообразий с пучком поверхностей дель Пещо // Изв. РАН. Сер. матем. 1998. Т. 62. № 1. C. $123-164$.

4. Corti A., Pukhlikov A., Reid M. Fano 3-fold hypersurfaces // Explicit birational geometry of 3-folds. Cambridge: Cambridge University Press, 2000. P. 175-258. (London Math. Soc. Lecture Note Ser. V. 281.)

5. Исковских В.А., Манин Ю.И. Трехмерные квартики и контрпримеры к проблеме Люрота // Матем. сб. 1971. Т. 86. №1. С. 140-166.

6. Pukhlikov A. V. Birational automorphisms of Fano hypersurfaces // Invent. Math. 1998. V. 134. № 2. P. 401-426.

7. Пухликов А. В. Бирационально жесткие двойные гиперповерхности Фано // Матем. сб. 2000. T. 191. №6. C. 101-126.

8. Pukhlikov A. V. Essentials of the method of maximal singularities // Explicit birational geometry of 3-folds. Cambridge: Cambridge University Press, 2000. P. 73-100. (London Math. Soc. Lecture Note Ser. V. 281.)

9. Pukhlikov A. V. Birationally rigid Fano complete intersections // J. Reine Angew. Math. 2001. V. 541. P. 55-79. 
10. Пухликов A. В. Бирационально жесткие итерированные двойныенакрытия Фано // Изв. РАН. Сер. матем. 2003. Т. 67. №3. С. 139-182.

11. Пухликов А. В. Бирационально жесткие расслоения Фано // Изв. РАН. Сер. матем. 2000. T. 64. C. 131-150.

12. Pukhlikov A. V. Certain examples of birationally rigid varieties with a pencil of double quadrics // J. Math. Sci. 1999. V. 94. №1. P. 986-995.

13. Пухликов $A$. В. Бирациональные автоморфизмы алгебраических многообразий с пучком двойных квадрик // Матем. заметки. 2000. Т. 67. № 2. С. 241-249.

14. Шафаревич И.Р. (ред.) Алгебраические поверхности. Сборник трудов семинара И.Р. Шафаревича // Труды МИАН. 1965. Т. 75.

15. Манин Ю.И. Рациональные поверхности над совершенньми полями. II // Матем. сб. 1967. Т. 72. C. $161-192$.

16. Манин Ю. И. Кубические формы. Алгебра, геометрия, арифметика. М.: Наука, 1972.

17. Исковских В. А. Рациональные поверхности с пучком рациональных кривых // Матем. сб. 1967. Т. $74(116)$. С. $608-638$.

18. Исковских $B$. A. Рациональные поверхности с пучком рациональных кривых и положительньм квадратом канонического класса // Матем. сб. 1970. Т. 83 (125). С. 90-119.

19. Исковских В. А. Бирациональные автоморфизмы трехмерных алгебраических многообразий // Итоги науки и техники. Соврем. пробл. матем. Т. 12. М.: ВИНИТИ, 1979. C. $159-235$.

20. Саркисов В.Г. Бирациональные автоморфизмы расслоений на коники // Изв. АН СССР. Сер. матем. 1980. Т. 44. № 4. С. 918-945.

21. Саркисов В.Г. О структурах расслоений на коники // Изв. АН СССР. Сер. матем. 1982. T. 46. № 2. C. 371-408.

22. Исковских В. А. О проблеме рациональности для трехмерных алгебраических многообразий, расслоенных на поверхности дель Пеццо // Труды МИАН. 1995. Т. 208. C. $128-138$.

23. Соболев И. В. Об одной серии бирационально жестких многообразий с пучком гиперповерхностей Фано // Матем. сб. 2001. Т. 192. № 10. С. 123-130.

24. Соболев И. В. Бирациональные автоморфизмы одного класса многообразий, расслоенных на кубические поверхности // Изв. РАН. Сер. матем. 2002. Т. 66. № 1. С. 203-224.

25. Гриненко M. М. Бирациональные автоморфизмы трехмерного двойного конуса // Матем. сб. 1998. Т. 189. № 7. С. 37-52.

26. Гриненко M. М. Бирациональные свойства пучков поверхностей дель Пещо степени 1 и 2 // Матем. сб. 2000. Т. 191. № 5. С. 17-38.

27. Гриненко М. М. О расслоениях на поверхности дель Пеццо // Матем. заметки. 2001. T. 69. № 4. C. 550-565.

28. Исковских В. А. О критерии рациональности для расслоений на коники // Матем. сб. 1996. T. 187. № 7. C. 75-92.

29. Corti A. Factoring birational maps of threefolds after Sarkisov // J. Algebraic Geom. 1995. V. 4. № 2. P. 223-254.

30. Sarkisov V. G. Birational maps of standard $\mathbb{Q}$-Fano fibrations // Preprint. Kurchatov Institute of Atomic Energy, 1989. 